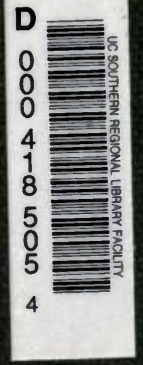

fornia

nal

ty 







\section{BOTANICA NEGLECTA}

\section{WILLIAM YOUNG, JR.}

(OF PHILADELPHIA)

\section{“BOTANISTE DE PENSYLVANIE"}

AND HIS

LONG-FORGOTTEN BOOK

BEING A FACSIMILE REPRINT

OF HIS

“Catalogue d'Arbres Arbustes

et Plantes Herbacees d'Amerique"

PUBLISHED IN PARIS IN 1783

With Prefatory Account of the Author and

Critical Notes by the Editor

SAMUEL N. RHOADS

PRIVATELY PRINTED, PHILAdelphia

1916 


\begin{abstract}
$\mathrm{F}^{\mathrm{F}}$ this First Impression of 250 Numbered and Signed copies, 50 are printed on large paper, quarto; the remainder, octavo, on buff-tinted rag paper; all uniformly bound in boards, uncut, paper label.
\end{abstract}

This is No. 


\section{PREFACE.}

Incredible as it may seem, in these days of literary research, the EARLIEST PUBLISHED BOOK, written by an American Botanist and devoted exclusively to American Botany, Horticulture and Floriculture, has been either purposely ignored or entirely overlooked by scientists, historians and bibliographers.

Dr. William Darlington, in his fascinating Memorials of John Bartram and Humphry Marshall, says of Marshall's "A rbustrum Americanum":- "This is believed to be the first strictly American botanical work-that is to say, the first treatise on American plants written by a native American and printed in this country."

But the Catalogue of William Young, Jr., herewith reprinted, while not originally printed in America, is, in other respects, in the same category with the Arbustrum, and antedates the latter by two years. It is interesting to note that Marshall knew of Young's Catalogue, and his reference to it on page 48 of the Arbustrum is the only one I have been able to find in all literature. Marshall, referring to the shrub "Fothergilla Gardeni" says:- "This, in some late Catalogues, has been called Youngsonia, in honour of William Young, Botanist, of Pennsylvania; but by Dr. Linnæus, Fothergilla, in honour of the late Dr. Fothergill, of London. It was first sent to Europe, from Carolina by John Bartram, to his friend P. Collinson by the title of Gardenia." The reader is referred to top of page 54 of this reprint for Young's version of this generic name, there claiming to have sent Dr. Fothergill a specimen in 1769, which was forwarded to Linnæus. Dr. Alex. Garden of Charleston, however, had previously sent it to Linnæus in 1765 , as noted by Sir J. E. Smith, under Garden's letter to Linnæus, dated May 18th of that year.*

The copy of William Young's Catalogue which forms the basis of this Reprint, first came to the editor's notice while looking over a price-list of old books, issued in August, 1915, by a dealer in Scotland.

It was obscurely listed as a sort of supplement or afterthought to the description of a copy of Marshall's Arbustrum Americanum, with which it had been rebound about 100 years ago. Contrary to my usual experience in ordering rare Americana from foreign Catalogues, I fortunately secured this item. It was in an old, half-calf binding, Young's Catalogue at the end, with autograph on title and marginal annotations in ink of John Barclay, who was no doubt the original

*Smith, J. E. Correspondence of Linnæus and other Naturalists. London, 1821. Vol. I, p. 319 . 
owner, and who probably was the same person mentioned in the Linnzan Correspondence as purchasing American plants from Marshall. Barclay's annotations, of which a specimen has been allowed to remain on page 7 of this Reprint, were made before the Catalogue was rebound and they have been shaved in the making up. The latest known owner of the book was John G. Howe Drummond, whose armorial bookplate adorns the front cover.

As already hinted, my surprise was great at finding no mention of Young's pioneer masterpiece in standard books of Botanical reference. More careful research and correspondence on the subject with several of the best libraries and authorities failed to discover any bibliographic allusion to such a work either under the French and German spelling of "Yong" or the English of Young. A canvass of the more important American Libraries has failed to discover a single copy of this book, and for this reason alone the editor feels justified in reproducing it.

As this copy appeared to lack a page or two at end, I wrote to the librarian of the National Museum of Natural History in Paris asking if they had a perfect copy and was much gratified to get the following courteous reply from the Librarian in Chief, together with a tranecript of the missing last page, 55 .

\section{Muséum d' Histoire Naturelle Bibliotheque,}

\section{S. N. Rhoads, à Philadelphie,}

Paris, 3 Septembre, 1915.

Cher Monsieur:-Voici les renseignements que je puis vous donner au sujet du "Catalogue d' arbustis," etc. de Yong, dout vous vue parlez dans votre lettre du 20 aout.

"Je ne sais pas si le "Catalogue" en question est rare dans les Bibliothèques europeennes, mais en tout cas i'l existe dans notre bibliothèque, et complet.

Je vous recopie la p. 55 (et dernière) . . .

Je n' ai pas trouve, jusqu à present, aucune indication sur la personnalité de M. Yong.

Veuillez agreer, cher Monsieur, l'assurance de ma consideration tres distinguée.

\section{DENIKER,}

Bibliothecaire en chef.

Being unable to get any information, either from reference books or from persons, here or abroad, as to the identity and personality 
of this "Botaniste de Pensilvanie," it was happily suggested to my memory that I had read, some years ago, two volumes of the Correspondence between Linnæus, John Ellis, William Collinson, Dr. Fothergill and others. As William Young's Catalogue contained references to his dealings with these men, I again carefully searched through this fascinating collection of botanical letters and was rewarded by finding there four references to Young, all of the greatest importance in identifying him and his botanical career. The title of this work has already been referred to and given in a footnote on a previous page of this Preface.

The first of these references is in Vol. I, page 512, in a letter of Dr. Alexander Garden, of Charleston, South Carolina, to John Ellis, of London, dated July 25, 1761, where he says:- "I have at last met with a man who is to commence nurseryman and gardener, and to collect seeds, plants, \&c., for the London market. He is a sensible, careful man, and has a turn for that business. He shall receive all the advice and assistance that I can give him. I must beg your interest in his favour; that you would bespeak what custom and commissions you can procure for him from your gardeners or nurscrymen or from any gentleman who may want what our province affords. He wants much to be acquainted with Mr. Gray and Mr. Gordon, at Mile-End; and I must beg that you would procure some commission from them to him. He is to employ his whole time in procuring whatever may be ordered.

"His name is Young, and any letters for him inclosed to me will be taken care of. I must beg that you would endeavour to inform me, on his account, what the prices of our several seeds are, or the value of young plants of Loblolly Bay, Azalea, Umbrella Magnolia, Beureria, Magnolia palustris, Halesia, Stuartia, and such like."

In a letter, dated March 21, 1760, Dr. Garden wrote Ellis that John Bartram was staying with him for a few days. There is little doubt that Bartram was later responsible for the introduction of William Young to Dr. Garden, Young being a near neighbor of Bartram, as the sequel will show.

The next reference to Young is on page 522 of the same volume, in another letter from Dr. Garden to John Ellis, dated November 19, 1764, as follows:- "Agreeably to your desire, I have spoken to Mr. Young, and given him your directions and my best advice so that I doubt not but his seeds and young plants will be good and his prices much lower."

The letter from Ellis to Garden, here referred to, is not published in this work, nor elsewhere, that I can find. 
Under date of "London, Jan. 14, 1770," page 571 of the same volume, occurs one of the most significant references to our unknown "Botanist of Pennsylvania." It is written in a letter from John Ellis to Dr. Garden, thus :- "I had a letter from Linnæus, with his respectful compliments to you. He longs much to hear from you. You have seen, no doubt, the Fly-trap or Dionaa muscipula, which Mr. Young, the Queen's botanist, brought over. It grows in North Carolina, and is much esteemed here. I have sent Linnæus the characters of it, which gave him infinite pleasure."

Before taking up further the subject of the "Queen's Botanist," I will now quote the last reference to William Young in Smith's Linnæan Correspondence. It occurs on page 73 of Vol. II of that work and is a much earlier reference to Young's discovery and exportation of the Venus Fly-trap to England. The letter is one from John Ellis to Mary, Duchess of Norfolk, and dated "London, Oct. 11, 1768," and confirms Young's statements on page 35 of this Catalogue regarding his discovery of the Dioncea. Ellis, after describing the "New Sensitive Plant," which he terms "one of the rarest productions of the vegetable kingdom," writes the Duchess; "A few of these plants were brought over from Philadelphia this summer by one Mr. Young who sold them to Mr. James Gordon, seedsman, Fenchurch Street, and Mr. Brooks, in Holborn. . . . This is an entirely new Genus. I have sent its characters to Linnæus, our father in botany, which I suppose he will adopt."

Let us now turn to the most fascinating and reliable work relating to the history of early American Botany and see what we can find about William Young. I refer to Dr. William Darlington's "Memorials of John Bartram and Humphry Marshall." Philadelphia, 1849. As hinted in the opening paragraphs of this Preface, Darlington was evidently ignorant of Young's Catalogue or he would have alluded to it in his historical Introduction to the "Memorials," and necessarily given it two years priority over Marshall's Arbustrum, as an American production.

Darlington's book helps clear up the mystery of the "Queen's Botanist."

A letter from John Bartram to Peter Collinson is the first reference (page 266) to that dignitary. "Sept. 23rd, 1764. Dear Peter: - . My neighbour Young's sudden preferment has astonished great part of our inhabitants. They are daily talking to me about him, that he has got more honour by a few miles traveling to pick up a few common plants than I have by near 30 years travel, with great danger and peril. It is shocking, the plants you have had, 
many of them known a hundred years, and most, twenty or thirty, should be esteemed at court as new discoveries. Several of my friends put me upon sending my new discovered specimens to the King, to try my success. Accordingly I have put up a little box of such specimens as I am sure he (Young) never found, and I believe never came to England before I sent them. The box I sent to thy care with a letter to the King under cover to thee which pray deliver to his Majesty."

Honest John was evidently a bit uneasy that his fresh young neighbor might supplant him in the favor of George the Third, and, under date of October the 16,1764, he writes (page 267):"Dear Peter: I sent by Captain Budden, by my neighbour Young, my spring specimens and a vial of Chinquapins, to try how they will do that way.

"Various are the opinions of Young's success. Some think he will make such an awkward appearance at court that he will soon come back again. Others that the Queen will take care of the German gentleman. I think that if he is put under Dr. Hill's care he will make a botanist, as he is very industrious and hath a good share of ingenuity.

"I hope thee will find some way to forward the box I sent to thee for the King, not that I depend on having any such preferment as Young had, but chiefly as a curiosity, to see what difference will be made betwixt such rare plants as never grew in Europe or Asia and such as have been growing in the English Gardens between twenty and one hundred years past, for such, I believe, were most that Young sent. But I and several others would be greatly pleased with a list of what he sent."

On April 9, 1765, Collinson wrote Bartram (page 268) that he had secured for him an appointment as "Botanist to the King" with a salary of $£ 50$ yearly, but making no reference to Young.

In May, 1765, he again writes and makes a brief allusion to the Queen's protégé, thus (page 270):- "I have not seen Young for some time. I conclude he is prosecuting his botanic studies."

With Collinson's next letter to Bartram on this topic (page 279), the plot considerably thickens and our interest kindles somewhat"May 28, 1766. My dear John, I wonder thou should trouble thyself about the Queen, as she has Young, and everything will be shown him. It cannot be expected he will favour any one's interest but his own. He is now so new-modelled and grown so fine and fashionable, with his hair curled and tied in a black bag, that my people, who have seen him often, did not know him. I happened not to be at home, so could not inquire what scheme he is upon." 
As we proceed with Dr. Darlington's book, we are rewarded, on page 285 , by a sudden shift in the drama. Bartram writes, Dec. 5,1766 , to his beloved "Peter"- "I am surprised that Young is come back so soon. He cuts the greatest figure in town, struts along the streets, whistling, with his sword and gold lace, etc. He hath been three times to visit me-pretends a great respect for me. $\mathrm{He}$ is just going to winter in the Carolinas; saith there is three hundred pounds sterling annually settled upon him. But Captain Chancellor tells odd stories of him: that he was put in prison, from which he was taken by two offieers and put on board ship: but his friends utterly deny it. Its pity but the truth was known, and the lying party snubbed."

Collinson's answer to this letter (page 286), written Feb. 10, 1767, completes the picture, and emphasizes the startling contrasts between the neighbor Botanists of Darby Road in "Old Kingsess"King's Botanist in Quaker homespun versus Queen's Botanist with lace and sword; honest, toil-worn, English John versus courtly, pampered, German William. Collinson writes:- "I believe there is too much truth in what the Captain saith about Young. He may live to repent his folly and extravagance. Such an opportunity lost is never to be regained unless he has better fortune than he deserves. As a friend, I advised him often to economy and industry, and not to sacrifice everything to his pleasures; for I foresaw, by his way of going on, how it must end; for I knew his salary could by no means support his expensive way of living."

The next word we have of Young in this book is to his credit and shows that he did not lack generosity, which, added to other good qualities attributed to him in his earlier career, probably wrought some reformation, as he eventually settled down to botanical and agricultural life on his father's farm at Kingsessing, very near Bartram's Garden. Bartram, in a letter to Dr. John Fothergill, had evidently accused Young of unfair treatment of him, but Fothergill wrote him (page 344) (no date, circa 1772?) :- "In a letter to my nephew thou intimates that probably Will Young may have endeavoured to raise some prejudice against thee. He has not. He durst not attempt it, as he knew my esteem for thee. He never spoke one word to thy disadvantage. . . . I have endeavoured to assist this poor man and have aided him considerably, but he will not succeed, nor can he be supported."

On page 504 of the "Memorials," Dr. Fothergill writes Humphry Marshall from London, 11th of Second Month, 1771:

“. . . . William Young sends his plants over very safely, by 
wrapping them up in moss, and packing them pretty close in a box. They come thus very safe, and we lose very few of them. He ties the moss in a ball about the roots with a piece of pack thread or matting, or hemp strings, and puts them so close as to prevent them from shaking about in the box."

Again (page 509) Fothergill writes Marshall:

"Ninth Month, 1772. . . . I had a plant of the great American Nymphra (Nelumbium) from W. Young. It put out leaves and the appearance of a flower, but did not flourish. I should be glad of another root, if it could be easily obtained. . . .

"I know not whether J. Bartram or any of his family continue to send over boxes of seeds as usual. He collected them with much care and they mostly gave satisfaction. W. Young has been very diligent, but has glutted the market with many common things; as the Tulip Trees, Robinias and the like. But, contrary to my opinion, he put them into the hands of a person, who, to make the most of them, bought up, I am told, all the old American seeds that were in the hands of the seedsmen here and mixed them with a few of W. Young's to increase the quantity. Being old and effete, they did not come up, and have thereby injured his reputation. I am sorry for him ; have endeavoured to help him; but he is not discreet."

Thus ends William Young's record from these sources, so let us now turn to others for such information as may be germane. In this search I have been much helped by my friend the well-informed historian of Germantown botanists and gardeners, Edwin C. Jellett, who had come across family records of the Darby Road Youngs.

William Young's father, also named William, not only owned the farm of $241 / 2$ acres where W. Young, Jr., lived, not far from J. Bartram's Garden, but also a plantation which he called "Blockley" in the same neighborhood. The Blockley Farm was his home. His will, the original of which I have examined at the Register's Office in Philadelphia, indicates a person of comfortable means and shows that in addition to agriculture he dealt extensively in, and probably manufactured, a patent medicine named "Hill's Balsam." Debts to him for this Balsam alone, in hands of his agents in the Colonies, were estimated at over 300 pounds sterling by his Executors. He makes no mention of his son in his lengthy will, but leaves his property to his widow and two married daughters and their children, and specially provides for $21 / 2$ acres in the centre of his Kingsessing property to be known as "William Young's Burying Ground." This graveyard yet exists, entirely surrounded by city houses, and is on Fifty-second Street, one square west of Darby Road. 
It is now locally known as the "Leech" or "Gaul" Burying Ground. The inscription on the founder's tombstone reads :- "In memory of William Young, who departed this life May 28, 1785, aged 72 years, 4 months, 14 days," etc.

Why the name of William Young, Jr., was not given in his father's will is explained by some on the ground that he died before his father. I have examined the original will of William Young, Jr., which was written at Kingsessing, December 4, 1784. It is quite lengthy, written entirely in his own hand, and signed "William Young, Jr."

The writing, spelling and style indicate a person of the average limited education of that period. He leaves the Plantation to his "wife Martha" and after her death it is to go to his "Boy Colly," who is to be "Larned to Read, \& Write and so must be send to Lern it," etc. Colly was probably named after Peter Collinson, Young's London patron, but it seems strange he did not call him his son, if, indeed, a son he was!

The junior Young, his will, was probated July 19, 1785, and in the absence of any record of his death, it seems doubtful that he died before his father, whose will was probated June 11th of the same year. It is probable that Young's father intended to disinherit him and that the son knew he had no tenure of nor right in the ground on which he was allowed to live.

The "Queen's Botanist" named as his Executors his wife and his two brothers-in-law, Leech and Hofman. The appraisement of his effects identifies him beyond question as our man. Witness these entries-"Sundry Plants brought from Carolina, £25." "Moveable Plants in Boxes, £22 10 sh."

It would be interesting to know the fate of "Martha" Young and her "Boy Colly" and whether descendants of that namesake of one of the greatest patrons of the Linnzan age of Botany may now be living.

Thus we know that "M. Yong, Botaniste de Pensylvanie," up to the time of his death, in 1785, continued to deal in the "Arbres, Arbustes (etc.) d'Amerique," named and described in his "Catalogue", which had been printed in Paris two years before. We have no record of the date of his birth, but from what we do know, he should have been in the prime and vigor of middle life when he died, a result, perhaps, of his extravagances at the Court of London and in the capitals of Europe.

Considered from a scientific standpoint, Young's Catalogue signifies very little; in fact, makes no pretensions. His nomenclature 
is neither binomial, nor trinomial, but a sorry mixture of the Brissonian, Catesbian and Linnæan systems.

He names many "New Species" and varieties, not from the standpoint of the systematist, but mayhap with the hope that this feature would tempt his patrons in France to increase their orders for specimens.

As stated in the Preface of his book, the object of Young's publication was to acquaint gardeners and collectors of American plants with the names of what were obtainable, their average size, general appearance and the kind of soil and amount of moisture required in their propagation.

Nevertheless, in these hair-splitting days of the indoor naturalist, we have no guarantee that some microscopic investigator will not take a copy of this Reprint to his den and thereby shake the very foundations of botanic priority! I have submitted the book to several of our most prominent systematic botanists and zoologists in the eastern United States and they all declare, that, from the systematic standpoint, it would be a great mistake to consider Young's so-called novelties seriously. In nearly all cases they are no more than nomina nuda, bare names, and, as such, cannot affect synonymy.

In other respects, however, Young's Catalogue will claim the interest of the antiquary and the historian. Resurrected from a life-long oblivion of 132 years, it now assumes a significant place in the early history of American Botany, and the meteoric career of its obscure author, from an amateur collector of seeds and plants under the guidance of Dr. Garden, to the post of Botanist to Queen Charlotte of England, serves to heighten our interest in his celebrated botanical contemporaries of the golden age of Bartram, Marshall, Collinson, Fothergill, Ellis and Linnæus.

S. N. Rrodds, Haddonfield, N. J., Feb. 1, 1916. 



\section{A T A L O G U E}

\section{D'ARBRES, ARBUSTES}

ET PLANTES HERBACÉES D'AMERIQUE,

Par M. YONG, Botanifte de Penfylvanie. PPan alay

$\mathrm{Ce}$ Catalogue eft divifé en deux parties; la premiere contient les Plantes que M. Yong peut fournir aux Européens, foit en graines, foit en plants.

La feconde contient celles qu'on ne pourra fe prosurer, qu'en les demandant dans d'autres Provinces.

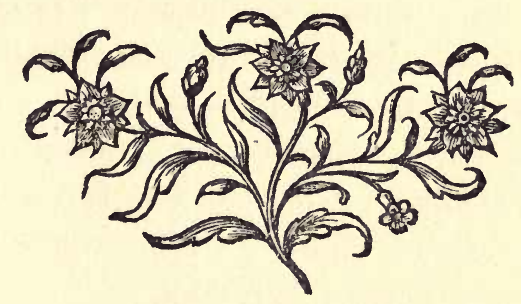

A P A R I S,

De l'Imprimerie de la V. Hér ISSANT, Imprimeur du Cabinet du ROI, Maifon \& Bâtimens de SA MAJESTÉ.

M. DCC. LXXXIII. 


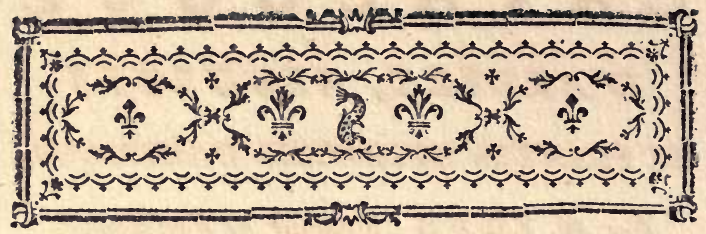

AVERTISSEMENT.

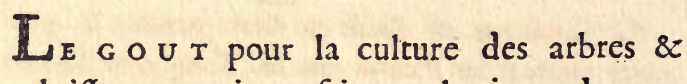
arbrifleaux exotiques fait tous les jours de nouveaux progrès. La plupart des Cultivateurs qui font affez heureux pour réuffir dans les femis. qu'ils font, au hafard, des graines de l'Amérique feptentrionale, placent les jeunes Elèves dans leurs jardins, fans connoître le terrein qui convient particulièrement aux différentes ef́pèces d'arbres, d'où réfulte la perte de la plus grande partie de ceux quils plantent. Les femis réuffiffent difficilement lorfqu'on ne les fait pas dans le terrein qui eft convenable aux différentes efpèces de graines.

Quelques Amateurs ont engagé M. Yong, Botanifte \& Marchand de plants \& graines en Penfilvanie, de faire un Catalogue des arbres, arbriffeaux \& plantes agréables dont il fait commerce, de la nature du terrein \& de l'ex. pofition qui convient à chaque efpèce. 
C'eft ce Catalogue qu'on met au jour pour la commodité \& l'utilité des Cultivateurs, ils y trouveront le double avantage d'être guidés dans le choix des terreins convenables aux femis \& aux plantations qui en réfulteront.

Ceux qui voudront faire des demandes à $M$. Yong, pourront s'adrefer à M. Villemorin, Succeffeur de M. Andrieux, Marchand Grenetier fur lc Quai de la Mégifjerie, iu Roi des Oifeaux, qui eft en correspondance ayec lui.

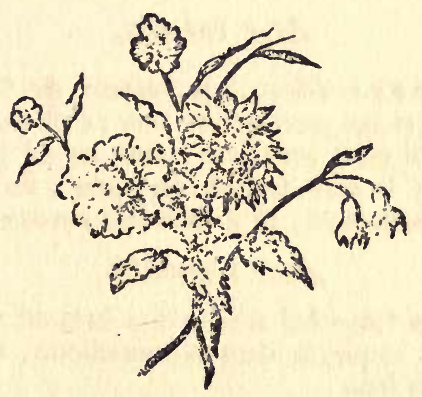




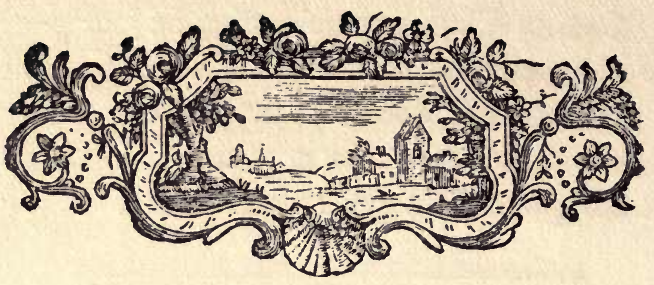

C A T A L O G U E $D^{\prime} A R B R E S, A R B U S T E S$ ET PLANTES HERBACÉES D'AMÉRIQUE.

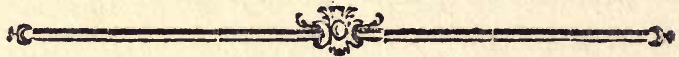

\section{ARBRES, ARBUSTES.}

\section{Acer rubrum.}

Cet a RB Re s'élève à la hauteur de 60 pieds; fon bois fert aux ouvrages de tour; c'et un bon bois à brûler; il croît lentement, aime un fol humide \& argilleux : fa sève fournit du fucre; fes femences périflent facilcment, \& il doit être envoyé en plant.

\section{Acer negundo.}

C'eft un très - bel arbre; fon bois eft très-beau, très-bon à employer dans la menuiferie, \& il aime les terreins frais.

$$
\text { Acer faccharinum. }
$$

Cet arbre parvient jufqu'd 80 pieds de hauteur, \&: à une groffeur furprenante. Il aime un fol riche \&: l'ger, fon bois eft bon à brûler, \& fournit du fucre; on m'a dit qu'un gros arbre peuit donner jufqu’a 100 


\section{(2)}

gallans de jus, qui, après avoir été bouillis, produilent environ 30 livres de fucre.

$$
\text { Acer fylveftre. }
$$

Cet arbre devient très-grand, \& d'une groffeur confidérable; il a depuis 60 jufqu'a 70 pieds de hauteur; c'eft un bon bois à brûler, il croît très-vîte dans un fol humide \& léger, fa. sève donne du fucre; fes femences ne produifent pas, fi elles ont été trop long temps hors de terre; les plants en font rares, mais ils doivent avoir la préférence dans les envois.

\section{Alnus pumila.}

Cet arbufte s'élève à 10 pieds dans un terrein riche \& hunide.

\section{Andromeda calyculata.}

Cet arbufte s'élève à 2 pieds de haut, il a de trèsbelles fleurs, \& il eft Jemper virens; il croît dans l'eau qui n'eft pas courante, mais il vient dans les terres grafles \& argilleufes; s'il eft tranfplanté, en le conlervant humide, il doit être envoyé en plant.

$$
\text { Andromeda lanceolata. }
$$

Cet arbufte s'élc̀ve à 6 pieds de haut, fes fleurs font belles; il vient dans un terrein humide $\&$ argilleux.

\section{Andromeda mariana.}

Cet arbufe a depuis un pied jufqu'à trois pieds de haut; il a de très-belles fleurs, \& il eft en fleur la plus grande partie de l'été; il vient dans un fol léger \& fablonneux, il eft très-long à venir de graine, \& doit être envoyé en plant.

\section{Andromeda Paniculata.}

Cet arbufte a depuis 2 jufqu'a 8 pieds de haut; il 


\section{(3)}

a de jolies fleurs, qui font placées en bouquet fur. le fommet de cet arbufte; il eft très - lent à venir de graine, \& doit être envoyé en plant.

\section{Annona folizs lanceolatis:}

Cet arbre a 20 pieds dé haut, fon fruit eft bon 2 manger; fon écorce eft forte; on en peut faire des cordes; il croît dans un fol riche \& léger.

\section{Aralia Spinofa.}

Cet arbufte croît jufqu'a 'Is pieds de haut; il fournit une gomme qui eft fort utile dans la médecine; il croît fur toutes fortes de fols.

$$
\text { Aralea altifima. }
$$

Cet arbufte a environ 8 pieds de haut, il a de belles fleurs, \& fleurit en mai; il vient dans un fol humide \& riche : il doit être envoyé en plant.

$$
\text { Azalea flore rojeo. }
$$

C'eft un très-bel arbufte à fleur; il fleurit en mai, croît dans un fol léger, a.environ 4 pieds de hauteur, \& doit être envoyé en plant.

$$
\text { Azalea nudiflora rubra. }
$$

C'eft un petit arbufte d'environ 8 pouces de haut; fes fleurs font élégantes, il flcurit en mai, aime un fol léger \& ftérile; il doit être envoyé en plant.

$$
\text { Azalea vifcofa nudifiora. }
$$

C'eft un petit arbufte d'environ 6 pouces de haut; il fleurit en mai, fon odeur eft agréable; il croît dans un fol léger \& ftérile, il doit être envoyé en plant.

$$
\text { Azalea vifcoja. }
$$

Cet arbufte a cuyiron 5 pieds de haut; fes flcurs 
font belles, d’une odeur agréable; il croît dans an fol argilleux, \& doit être envoyé en plant.

\section{Betula dilcis, nova Species.}

C'eft un très-bel arbre, qui devient très-grand \&e droit, \& parvient a la hauteur de 60 pieds; il poffede des grandes qualités médicinales, \& croît dans toutes fortes de fols.

\section{Betula lenta.}

Cet arbre n'eft pas très-gros, $f_{a}$ hauteur ordinaire eft de 40 pieds; il vient dans un fol riche, fablonneux \& humide.

Bignonia catalpa major.

Cet arbre croit de 40 à 50 pieds de haut; les gouffes qui renferment fes graines ont fouvent 12 pieds de long; fon bois eft tendre, \& vient très-vîte dans un. fol fablonneux.

\section{Bignonia catalpa minor.}

Cet arbre croît dans un fol fablonneux.

$$
\text { Bignonia crucigera. }
$$

C'eft une liane qui s'elève en grimpant autour des arbres, fuifqu'a la hauteur de 40 pieds; fa fleur eft très-belle \& monopéta'e : cette liane croît dans toutes fortes de fols.

$$
\text { Bignonia femper virens. }
$$

Cette liane monte fur les arbres jufquià 60 pieds de haut; clle a de belles fleurs, qui font monopétales, \& croît dans toutes fortes de fols.

\section{Carpinus lenia.}

Cet arbufte a Is pieds de haut, fon bois eft dus il croit dans un fol humide \& pierreux. 
Carpinus Virginiana.

Cet arbre croît depuis 20 jufqu’a 30 pieds; il eft lent dans $f_{a}$ croiffance, fon bois eft dur, \& il profite dans un fol riche \& humide.

\section{Ceanothus Americana.}

Cet arbriffeau croît aे 2 pieds de haut; on en fait un thé fain \& agréable; les Américains les plus fenfés le préfèrent au thé de la chine.

\section{Celaftrus bullatus.}

Il s'elleve de Is à 20 pieds de haut. II eft d'un grand ornement parmi les arbuftes, quand les cofies qui renferment fes femences mûriffent ; il croît dans toutes fortes de fols.

\section{Celaftrus fcandens.}

Cette liane s'ellève fur les arbres à la hanteur de 40 pieds. Son fruit eft médicinal \& pare les jardins. Elle croît dans un fol riche \& léger.

\section{C.fitis Occidentales.}

Cet arbre s'élève de 30 a 60 pieds de haut; il aime un fol léger; fon bois n'eft d'aucune conféquence.

\section{Cephalanthus Occidentales.}

Cet arbufte a 8 pieds de haut; il a de belles fleurs, $\&$ aime un fol riche $\&$ humide.

\section{Cerafus Canadenfis.}

Cet arbre a jufqu'd 60 pieds de haut, \& a 1 d 2 pieds de diametre. Il elt beau \& croît fort vîte daas toutes fortes de fols. 


\section{Cercis Canadenfis.}

Cet arbre a environ 20 pieds de haut; fes fleurs font très - belles, \& il vient dans un fol léger.

\section{Cercis pumila nova Species.}

Les fleurs de cet arbufte font d'un rouge foncé; il aime à croître parmi les rochers \& les pierres.

\section{Chionanthus Virginica.}

Cet arbre, ou plutôt cet arbufte, croît de ro d 20 pieds de haut. Quand il eft en fleurs, il eft trèsbeau; il profpère dans un fol riche $\&$ humide, mais fi fes racines font bonnes, il vient bien dans toutes fortes de fols.

\section{Clethra alnifolia.}

Cet arburte a environ 5 a 6 pieds de haut;, fes fleurs font d'une odeur agréable. Il croît dans un fol léger, \& réufit dans tous les terreins où il eft tranfplanté.

\section{Clethra flore rubro.}

C'eft un arbufte affez rare; il a environ 3 pieds de haut, croît dans un fol léger, fes fleurs font couleur de pourpre.

Cornus cartice rubro.

Cet arbutte a deptris 5 jufquà 8 pieds de haut. It mérite d'être cultivé à caufe de lufage quion en peut faire, pour en treffer de forts paniers. Il croît dans un fol humide, riche \& léger, \& lorfquil eft deftiné pour cet ufage, on doit le couper jufquà terre chas que année.

\section{Cornus Floridanus.}

Cet arbre eft un ornement des jardins, quand il 
(7)

elt en fleurs; il s'ellève de 20 aे 30 pieds. J’ai guéri des fièvres intermittentes avec fon écorce. Il croit fur tous les fols.

\section{it coryius cornuta. Hoagle Mute}

Cet arbufte a 5 pieds de haut; fes noix font bohnes anger; il croît dans un terrein pierreux.

Crategus glauca, nova Species. Nesurcofthe

Ceft un arbufte d'environ 8 pieds de haut; fes épines font longues \& épaiffes, \& il croît fur toutes fortes de fols.

\section{Crategus nudo flore.}

Cet arbriffeau a trois pieds de haut; il vient dans un fol pierreux, \& il eft très-beau.

* Cratogus prunifolia, nova Species. Hock kitur

Cet arbuite a is pieds de haut, il eft épineux, a de jolies fleurs, \& croit fur toutes fortes de iols.

\section{Cratagus $V$ irginica major.}

Cet arbre a $20 \$ 25$ pieds de haut; fon fruit eft bon a manger, \& de la grofleur d'une cerife : il lui faut un fol riche \& léger.

\section{Crazagus vulgaris.}

Cet arbre a 20 pieds de haut; il eft plein d'épines, \& croit fur toutes fortes de fols.
y
Cupreffus thyoides.

C'eft un bel arbre toujours verd; il a 60 pieds de haut. Il vient vîte \& haut dans ún fol marecageux fur un fond de fable, \& lorfquil eft tranfplante jeure, il croît fur toutes fortes de terreins.

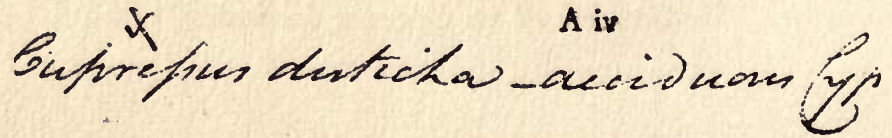




\section{(8) \\ Diofpiros dulcis:}

Cet arbre croît a la hauteur de 30 pieds; fon fruit eft le meilleur de toutes les efpèces; il croît dans un fol léger.

\section{Diospyros Virginica.}

Cet arbre parvient a 50 pieds de hauteur; fes fruits font doux \& d'un goût agréable dans leur mâturité, qui eft en Décembre \& Janvier, ils font de la groffeur d'une petite pêche; on en fait de l'eau-de-vie.

$$
\text { Dirca paluftris. }
$$

Cet arbufte a environ 3 pieds de haut: fon bois eft compact; les Botaniftes le regardent comme un arbrifleau curieux; il fleurit en Avril.

\section{Evonymus Americana.}

C'éft un très-bel arbrifieau de 6 a 8 pieds de haut; toujours verd, pour peu qu'il foit garanti du froid : fes-femences, quand elles font mûres, font un trèsbrillant effet; il aime un fol riche \& léger, \& peut être envoyé en plant.

$$
\text { Fagus Americana: }
$$

Cet arbre eft très-grand, \& il a jufqu’d 60 pieds de haut; il ent bon à bruller.

$$
\text { Fagus dulcis. }
$$

Cet arbre devient très-haut, \& croît vite à la hauteur de Go pieds ; fes graines font d'une odeur agréable: il croît dans un fol itérile.

$$
\text { Fagus pumila ou chinquapin. }
$$

Cet arbufte a depuis 4 jufqu'à Is pieds de hatt; il porte un frut très-agreable cn forme de gland; il eft curieux \& croit dans un terroir Atérile: 
Fraxinus juglandi-folia alba:

Ceft un bel arbre; il croit dans un fol léger environ 50 pieds de haut.

Fraxinus juglandi-folia nigra.

Cet arbre devient grand, \& sélève a la hauteur de 80 pieds fur un fol riche \& humide; fon bois eft bon pour le charronnage \& pour brûler.

\section{Gualtheria procumbens.}

Ceft un très-bel arbriffeau, qui n'a guere que 2 ou 3 pouces de haut; il eft femper virens : on fait de fes feuilles un thé agréable \& fain; il croît dans un fol leger, \& il faut lenvoyer en plant, plutôt qu'en graine.

\section{Gleditschia triacanthos.}

Cet arbre croît de 40 a 60 pieds de haut; fon écorce eft médıcinale ; fon bais eft bon pour faire des chevilles employées dans la bâtiffe des vaiffeaux, parce quili eft très-dur \& durable; il crôt dans un fol fa. blonneux \& léger.

\section{Hamamelis latifolia.}

Cet arbre s'elève de 8 a ro pieds; c'eft lunique efpèce de ce genre connue dans la Botanique; il aime un fol léger \& pierreux.

\section{Hedera quinquefolia.}

Cette vigne-vierge grimpe fur les arbuftes a la hauteur de ro pieds, \& rampe fur la terre à quelque diftance; elle eft tres-belle pour tapiffer les murs, \&c.

Hypericum canaliculatis foliis.

C'eft un très-bel arbufté a fleurs; il croît de 2 aे 4 
pieds de haut, \& fleurit en Août; il eft très-difficile de le propager de femence.

$$
\text { Itea Virginica. }
$$

Cet arbufte acquiert environ 6 pieds de haut dans un fol riche \& hunide.

$$
\text { Juglans alba aquatica. }
$$

Cet arbre devient très-grand, \& s'èlève à 70 pieds de haut; fon bois elt dur, compact \& bon aे brûler; il croît dans un fol hunide \& léger.

\section{Juglans alba cortice hirfuto.}

Cet arbre eft haut \& droit, il a jufqu'a 70 pieds; fon bois eft dur \& compact, fes noix font les meilleures $q u i l y$ ait en Amérique. Leurs coquilles ne font point dures; elles ont un goût d'amande, leur odeur eft agréable; il vient dans un fol riche \& humide.

\section{Juglans alba dulcis.}

Cet arbre croît à 60 pieds de haut; fes noix font douces \& bonnes à manger, leurs coquilles font dures, le bois eft dur aufi \& compact, \& bon à brûler; il croît dans un terrein riche \& léger.

$$
\text { Juglans alba foliis ovatis. }
$$

Cet arbre croit de 30 à 50 pieds de haut; fon bois eft dur \& compact; il vient bien dans un fol riche \& léger; c'eft un bon bois a brûler.

$$
\text { Juglans alba fructu cordato: }
$$

Ce noyer n’eft pas fi gros que les autres efpèces; fon bois eft caffant, fes noix font tendres \& ameres; il donne un bon bois a brûles, \& croît dans un fol riche \& humide. 


\section{(II)}

\section{Juglans alba fructu pinnato:}

Cet arbre eft grand, \& croît 270 pieds de hatut ; fon bois eft dur \& compact, \& propre à tous lés ouvrages qui exigent ces fortes de qualites. Mais il ne doit pas être expofé à l'air, car l'humidité le pourrit bicntôt; c'eft un excellent bois à brûler; il croît dans un fol riche, humide \& léger, mais lentement; fes noix font bonnes à manger, \& leurs coquilles font dures.

\section{Juglans alba major.}

Cet arbre eft grand \& fort, fa hauteur eft d'environ 60 pieds; fon bois eft dur \& d'un grand fervice pour les charpentes des moulins, c'eft le meilleur bois à brûler quili y ait dans le monde; il croît lenternent, \& il äme un fol riche \& léger.

\section{Juglans alba non-fana.}

Cet arbre s'elève à environ 40 pieds de hauteur; fon bois eft dur, compact '\& très-bon à brûler; il aime un fol riche \& léger; il croît lentement, fes noix font pernicieufes.

$$
\text { Juglans alba Serratifolia. }
$$

Cet arbre croît a environ 60 pieds de haut, fon bois eft dur \& compact; il aime un fol riche \& leger, \& croît lentement.

$$
\text { Juglans foliis cordatis. }
$$

Cet arbre croît de 40 aे 50 pieds de haut. Il eft très-beau, fes noix font bonnes aे manger, donnent beaucoup d'huile : il' vient très-promptement dans un fol riche \& léger.

$$
\text { Juglans nigra major. }
$$

Cet arbre acquiert jufqu'a 60 pieds de haut, \& 2 
(12)

ou 3 de diametre. Il eft très-beaú, \& croît très-víte dans un fol humide \& riche; les coquilles de fes noix donnent une teinture d'une très-belle couleur brune.

\section{Juglans nigra major.}

Cet arbre s'elève a 60 pieds \& plus, \& il a un pied de diamètre; fes noix font bonnes à manger, \& donnent beaucoup d'huile, fón bois fait de trèsbeaux meubles; il croît très - promptement dans un fol riche \& humide.

\section{Juniperus Phanicoa:}

C'eft un bel arbre d'environ 20 pieds de haut; il croît dans un fol léger.

\section{Juniperus Virginiana:}

Cet arbre croît très-vîte jufqu’a so picds de haut, dans un fol riche \& léger; mais, dans un terrein ftérile \& fablonneux, il ne parvient qu’à 20 ou 30 pieds de haut, \& cependant le fable elt fon terrein ordinaire ; fon bois eft étonnermment durable : jen ai vu employé en clôture, q̧ui fubfintoit depuis 80 ans dans la terre, \& qui, felon toute apparence, étoit encore très-bon.

$$
\text { Kalmia anguftifolia. }
$$

C'eft un très-bel arbufte à fleurs; il a environ un pied de haut, croît dans un fol fablonneux \& humide, vient difficilement de femence, \& doit être 'envoyé en plant.

\section{Kalmia glauca.}

C'eft un très-bel arbufte à fleurs; it a environ tun pied de haut; il aime un fol riche \& léger, il eft 
long a croître de graine, \& il doit être envoyé ex planto

\section{Kalmia latifolia.}

Cet arbufte eft très-beau; il eft toujours wert; il fleurit en Juin, \& fes fleurs font charmantes, fes feuilles font d'un qualité pernicieufe; il croît depuis 2 jufqua.a is pieds, mais il eft difficile de le faire venir de femence. $J^{\prime}$ ai vu des plants, en Angleterre, dans la pépiniere de M. Gordon, quili me dit avoir femé il y avoit quinze ans, \& qui navoit encore que quatre pouces de hauteur, \& par conféquent ils doivent être envoyés par plant : il croît fur un fol léger.

\section{Kalmia tenuifolia.}

C'eft un très-bel arbufte toujours vert; il a environ un pied de haut, fes fleurs font délicicufes; il croît dans un fol fablonneux, il aime l'humidité, \& il eft bon de l'envoyer en plant.

\section{Laurus aftivatis.}

Cet arbufte croît d'environ 6 pieds de hait dans' un fol riche \& humide; il fleurit en Avril ; fes femences périffent quand on les garde, \& il doit être envoyé en plant.

\section{Laurus Saßafras.}

Cet arbre eft bien connu par fes qualités médicinales; il s'élève de 20 à 25 pieds de haut dans un fol riche \& léger; mais fes femences, en général, ne fe confervent point, \& il faut l'envoyer en plant.

\section{Liriodendron tulipifera.}

C'êt le plus grand arbre d'Amérique \& de tout Te monde; il acquiert jufqua à 80 pieds de hauteur; il eft dune grofleur étonnante, croît très-promptcment 


\section{(14)}

Iur toutes fortes de fols; fon bois eft d'un grand fervice dans les bâtimens; il lui faut un fol léger, riche \& humide \& il eft communément mâle.

$$
\text { Liquidambar aspleni folia. }
$$

C'eft un bel arbriffeau qui s'élève à environ 3 pieds, fes fleurs ont une odeur très - agréable; il croît dans un terrein léger \& fablonneux, \& doit être envoyé en plant.

$$
\text { Liquidambar ftyraciftua. }
$$

Cet arbre s'élève jufqu’a 60 à 80 pieds de hauteur; il fournit une gomme médicinale, \& ne croît pas très-promptement; fon bois fait de très-bons ouvrages d'ébéniftrerie; il croît dans un fol humide \& argilleux.

\section{Liquidambar fyraciflua ligno rubro; nova species.}

Ceft un grand arbre qui sélève jufqu'à 70 pieds de haut, \& qui a environ deux pieds de diametre; fon bois eft très-beáu \& varié de veines rouges, on en fait des meubles délicieux; il croît dans un fol léger.

$$
\text { Lonicera marylandica. }
$$

Cet arbufte a environ 6 pieds de haut, fleurit en Mai, \& vient fur toutes fortes de fols.

$$
\text { Lonicera Virginica. }
$$

Cet arbriffeau s'élève à is pieds de hauteur, \& fouvent il eft foutenu par les arbuftes voifins; il croît dans un fol fablonneux.

\section{Magnolia glauca.}

Cet arbre croît de ro à 20 pieds de haut, fes Reurs font les plus odoriférantes de tous les Magnolia; 


\section{(is)}

Il eft employé dans la médecine; il croît dans un fol riche \& humide; cependant je l'ai vu venir très-heureufement, en Angleterre, fur un fol de terre graffe \& argilleufe : il faut l'envoyer en plant.

\section{Malus Virginica.}

Cet arbre parvient $\mathrm{d}$ environ 3 pieds de haut; fes fleurs font les plus belles, \& de l'odeur la plus agréable de toutes, parmi toutes les efpèces; fon fruit eft dur \& aigre, \& il vient bien fur toutes fortes de fols.

\section{Mespilus arborea.}

Cet arbre a 30 pieds de haut; fes fruits font excellents a manger, fon bois eft tendre; il croît dans un fol riche \& argilleux.

$$
\text { Mespilus, nova Species. }
$$

Cet arbufte croît de 2 a 8 pieds de haut; il a de belles fleurs pourpres; il croît dans un fol-riche \& humide.

$$
\text { Mespilus ferratifolius. }
$$

Cet arbriffeau a environ 6 pieds de haut; fes fleurs font belles; il croît dans un fol humide \& riche.

\section{Morus Virginica rubra.}

Cet arbre s'elève a environ 40 pieds de haut, fon fruit eft gros, \& il croît dans un fol riche.

$$
\text { Myrica foliis lanceolatis. }
$$

Il eft femper virens \& très-beall; il s'ellève à 4 pieds de haut, croît dans un fol ftérile \& Sablonneux; on fait une efpèce de cire de fon fruit.

$$
\text { Nyda aquatica. }
$$

C'eft un très - grand arbre qui s'élève jufqu'a 70 pieds 
de haut, fon bois eft d'une grande utilitt $\mathrm{a}$ caule de $f_{2}$ dureté; il vient fur un fol humide \& argilleux.

$$
\text { Ny』a montana. }
$$

Cet arbre a environ so pieds de haut; fon bois eft compacte; il croît. dans un fol léger.

\section{Pafiflora Virginica.}

Cette liane monte fur les arbres jufquá 20 pieds de haut; fes fleurs font petites : elle croit dans un fol riche \& léger.

\section{Pinus foliis geminis śrevioriöus.}

Cet arbre monte fort haut $\&$ fort vite dans un fol fablonneux; il a environ 70 pieds de hauteur.

\section{Pinus foliis geminis cono fquammofo.}

Cet arbre a environ 50 pieds de haut; il eft beau \& croit dans un fol fablonneux.

\section{Pinus foliis longioribus tenuioribus ternis.}

Cet arbre croît haut \& vîte dans un fol fablonneux; fouvent a la hauteur de 80 pieds \& de 2 à 3 de diamètre; il eft fort utile dans les bâtiments.

$$
\text { Pinus foliis fabris. }
$$

C'eft un très-grand arbre; il parvient jufqu'à 100 pied's de hauteur, on en fait de bons mâts; il croît très-vîte , \& n'a befoin que d'un fol léger.

\section{$P$ inus foliis ternis.}

Cet arbre reft pas fort gros; il a 30 \& 40 pieds de haut, \& il aime. un fol fablonneux.

Pinus foliis ternis, 


\section{(17) \\ Pinus folits ternis, conis longioribus fquam.

$$
\text { mofis. }
$$

Cet arbre vient très - grand \& tres - vîte dans un fol fablonneux; il a plus de 80 pieds de haut, fon bois eft utile pour les bâtimens \& les nâtures.

Pinus foliis ternis, conis longioribus fquammofis, minor.

Cet arbre croît de la hauteur de ro pieds, mais il eft en général mince; il croît plus vite dans un fol fablonneux que dans un autre terrein.

\section{Pinus Frankincea.}

Cet arbre n'eft pas fort gros, il a de 40 d 50 pieds de haut, c'eft un bon bois de charpente; il vient trèsvîte dans un fol fablonneux.

\section{Pinus heterophylla.}

Cet arbre rieft pas auffi gros que beaucoup d'autrcs efpèces; il s'élève' à la hauteur de 50 pieçs \& n'en á pas plus d'un de diametre; il croit fur un fol fablonneux.

$$
\text { Pinus kezerophylla, minor. }
$$

Cet arbre eft fort beau, a 30 pieds de haut, aime un fol fablonneux.

$$
\text { Pinus zeda. }
$$

C'eft un très-gros arbre, il s'élève jufquà 80 pieds de haut, donne un beaù bois débité en planches ou pour des mâts; il croît fur toutes fortes de fols.

$$
\text { Pinus tenuifolia. }
$$

Cet arbre devient très-gros, parvient a environ 80 pieds de haut, croît très-vîte \& dans un fol fablonneux 


\section{(18)}

\section{Pinus Virginiana, pralongis foliis senuioribus.}

Cet arbre n'eft pas fort grand, il n'a guere que 30 ou 40 pieds de haut, mais c'eft un arbre d'une tríso belle apparence; il aime un fol fablonneux.

\section{Platanus occidentalis.}

Cet arbre a jufqúà $60 \& 70$ pieds de hauteur $\&$ une prodigieufe groffeur; il doit être recommandé dans tous les pays où le bois à brûler eft rare, 'parce quỉil croît très-vîte, il eft aifé à couper \& donne un très-bon bois à brûler.

\section{Prinos glaber.}

C'eft un trìs-bel arbufte, toujours verd, qui s'elève de 2 à 6 pieds de haut; il croît dans un fol riche, husmide \& fablonneux.

\section{Prinos verticillatus.}

C'eft un très-bel arbufe, à caufe de fes bayes dont il eft paré , \& qui durent une grande partie de l'hiver; il crôt de 4 à 10 pieds de hatit; il aime un fol riche \& humide.

\section{Prunus Americana.}

Cet arbie s'elève à cnviron 20 pieds de hauteur, fon fruit eft jaune 2 aliez bon; il croît dans toutes fortes de terreins, mais fon terrein natif eft riche \& humide.

$$
\text { Quercus alba, major. }
$$

C'eft un arbre de 70 picds de haut; fon bois eft de grand fervice \& durable; il lui faut un terrein argillerx. 


\section{(19) \\ Quercus alba, minor:}

Cet arbre a environ 30 \ 40 pieds de haut, \& fon bois eft compact; il croit dans un fol humide $\&$ ar: gilleux.

$$
\text { Quercus efculus. }
$$

Cet arbre eft haut d'environ 60 pieds, en a trois de diametre; fon bois eft bon pour la charpente \& beaucoùp d'autres ufages, \& eft très-bon à brûler; il vient tres-vite dans un fol fablonneux.

$$
\text { Quercus efculus alba. }
$$

Ceft un grand arbre de 70 pieds de haut; il vient dans un fol argilleux, \& il eft bon pour la charpente.

\section{Quercus efculus alba, minor:}

Cet arbufte a de 3 \& 5 pieds de haut, \& croît dans un fol fablonneux,

Quercus efculus nigra, minor.

Cet arbufte croît à environ 6 pieds de hatt dans un fol fablonneux.

$$
\text { Quereus major. }
$$

Cet arbre eft le plus grand chêne connu; il a jufqu'à 70 pieds de haut; ceft un bon bois pour les conftructions \& pour braler; il aime un fol riche \& léger.

$$
\text { Quercus nigra; major. }
$$

Cet arbre a 70 pieds de haut; c'eft un bon bois de confruction; il creit dans un fol riche \& léger.

\section{Quercus nigra, minor.}

Cet arbre a environ 50 pieds de haut; c'eft un bois bon à brûler; il croît dans un fol leger. 


\section{(20) \\ Quercus pumila:}

Cet arbufte a depuis I pied jufqu' 5 de haut; it eft fi chargé de glands, que fouvent les branches courbent jurfqua terre fous leurs poids; il croît fur un fol ftérile. On trouve fouvent en Amérique des centaines d'acres de terrein qui font couverts de cet arbriffeau, \& qui fournifient de la nourriture \& un abri dे un grand nombre de quadrupèdes.

$$
\text { Quercus rubra, major. }
$$

Cet arbre s'élève jufqu'd 70 pieds; il eft bon pour la conftruction des bâtimens, \& croît dans un fol riche \& légeŕr; fon écorçe eft employée avec fuccès pour les fievres intermittentes.

Quercus rubra, minor.

Arbre de 60 pieds de haut, qui fert a beaucoup d'ufages \& croît dans un fol humide \& argilleux.

$$
\text { Quercus Salicifolia. }
$$

Cet arbre a 60 pieds de haut; il eft très-beau \& yient bien dans un fol humde \& argilleux.

\section{Rhododendron maximum.}

Cet arbufte eft trìs-beau; il eft toujours verd, fes fleurs font fuperbes; il a jufqu'a is pieds de haut; il eft trop lent à croître de lemence \& doit être envoyé en plant.

\section{Rhus copallinum.}

Cet arbriffeau croît à huit pieds de haut, dans un terrein fablonneux; fes fruits font aigres \& même corrofifs.

Rhus foliis pinnatis integerrimis, linnai.

Cet arbre croît de 10 a is pieds de haut \& fur toutes fortes de fols. 


\section{Rhus pumilum.}

Cet arbufte a environ 2 pieds de haut; il eft tres: beau \& croît dans un fol léger.

\section{Rhus ferratifolium.}

Cet arbufte a environ ro pieds de haut; on s'en fert pour teindre en noir; il croît dans un fol riche \& léger, une infufion de fes femences guérit un mal de gorge opiniâtre.

\section{Rhus vernix.}

Cet arbufte a depuis 5 jufqu dे 15 piéds de haut; if eft vénéneux; il lui faut ordinairement un terrein riclze $\&$ humide, mais fi fes racines font fortes, il profite dans toutes fortes de fols.

\section{Robinia hispida:}

Cet arbre ne devient pas très-grand; il n'a guere plus de 6 a 15 pieds de haut; fes fleurs font très: belles; il aime un fol léger.

\section{Robinia pseudacacia.}

Cet arbre a 40 \& 50 pieds de haut; il a de belles fleurs, fon bois eft d'un grand fervice pour faire des chevilles pour les vaiffeaux, parce quili eft très-dur \& durable; il croît dans un fol léger.

$$
\text { Rofa corymbofa. }
$$

Il donne de très-belles rofes; fes fleurs ont une odeur très-agréable, croiffent fur le fommet de l'ar. briffeau; elles font d'un beau rouge; elles durent tout lhiver \& ont la plus belle apparence; il vient dans un terrein humide, 


\section{(22) \\ Rofa foliis dulcibus.}

Ce rofier s'élève à to pieds de haut ; fes feuilles font douces; il vient dans un fol léger.

$$
\text { Rosa penfylvanica. }
$$

Ce-rofier a jufqu’à deux pieds de haut; il eft trèsbeau \& en fleur pendant 3 mois de fuite; fes femences végétent rasement, \& il doit être envoyé en plant.

$$
\text { Rubus procumbens. }
$$

Cette ronce a environ ro pieds de hauteur, \& trèslouvent elle rampe fur lá terre; fon fruit eft le meillieur de toutes les efpèces connures; elle aime un fol argilleux.

$$
\text { Rubus fricto flore, corymbofo. }
$$

Il a environ Io pieds de haut; fon fruit eft fort gros \& bon, \& il croit dans un fol riche.

\section{Sambricus Americana.}

Cet arbufte sélive à I 2 pieds de hauteur; fon écorce \& fes fleurs font employées en médecine; il vient bien dans un fol humide \& raiche.

$$
\text { Salix penfylvanica. }
$$

Cet arbutte a environ 2 pieds de haut; il eft fort joli, vient fur un terrein argilleux \& doit être envoyé en plant.

Sapin. $V$. Spruce.

Smilax lanceolatis folits, nova Species.

Ceft une très - belle efpèce de fimilax nouvellement découvertc; il a 60 pieds de haut, monte fur les arbres, $\&$ vient dans un terrein riche \& humide. 


\section{(23)}

\section{Smilax latifolia:}

C'eft une liane épineufe qui grimpe autour des arbres a la hauteur de 20 pieds; fes racines font employíes dans la médecine; elle croît dans un fol riche \& humide.

$$
\text { Spirea corymbofa. }
$$

C'eft un arbufte qui a jufqu’a ro pieds de haut, qui 2 de belles fleurs \& qui aime un fol riche \& humide.

$$
\text { Spruce balfamea, Abies. }
$$

Cet arbre a jufqu'a 60 pieds de haut \& environ 2 pieds de diametre; il eft beau, toujours verd, croît dans un fol léger \& parmi des roches.

\section{Spruce noir de terre neuve.}

Nota. Si on demande cet arbre, il faudra le demander fous le nom de Black New foundlond Spruce.

Cet arbre eft très-beaul \& s selelève $\grave{2} 60$ pieds de haut; Il fert à faire de la bière, \& on en fait des mâts pour de pctits bâtimens; il croît dans un fol léger \& humide.

\section{Spruce rouge de terre neuve.}

Nota. Si on demande cet arbre, il faudra le demander fous le nom de Rednew foundlond Spruce.

Cet arbre eft très-beau; croît a 60 pieds de haut; on fait de la bière très-faine de fon écorce, \& on fait des mâts de cet arbre pour les petits vaiffeaux; il croît dans un fol humide \& léger. 


\section{(24) \\ Staphylaa Serratifolia.}

Cet arbufte croît à environ 8 pieds de haut fur toutes fortes de fols.

\section{Tilia Americana.}

Cet arbre croît très - vîte \& d'une grande proportion dans un fol léger \& humide; il selève de 60 \& 70 pieds; fon bois n'eft point dur.

\section{Taxus procumbens.}

C'eft un très - bel arbufte, , femper virens; il a depuis 3 jufqu'à $s$ pieds de hauteur dans un fol pierreux.

$$
\text { Toxicodendron fraxini.folium. }
$$

Cet arbutte s'élève autour des arbres \& des arbuftes à la hauteur de 6 \a 10 pieds; il eft vénéneux, mais il eft d'un très-grand fervice pour la teinture en noir.

\section{Ulmus Americana.}

C'eft un - très grand arbre qui a 60 pieds de haut; il aime un fol humide \& léger.

$$
V \text { accinium album, acidum. }
$$

Cet arbufte croît a environ la hauteur de 8 pieds; il eft très-beau, fes fruits \& fes feuilles font etonnamment aigres, mais lorfque le fruit devient mûr, il eft bon à manger; cet arbufte croît dans un terrein grav veleux \& humide, \& doit être envoyé en plant.

$$
V \text { accinium corymbofum. }
$$

Cet arbufte a rarement plus d'un pied de haut; il eft très - beau; fes fruits font bons a manger, mais en petit nombre; il croît dans un terrein leger \& gra* veleux, \& doit être envoyé en plant. 
Vaccinium glaucum, nova Species.

Cet arbufte a depuis 2 jufqu'a 5 pieds de hatu: fes fruits peuvent fe manger, fans être d'un goût agréable; fes fleurs font jolies \& viennent en grand nombre; il croît dans un fol fablonneux \& graveleux; il peut être envoyé en plant.

\section{$V$ aciinium globularia folio, nova species.}

Ceft un très-bel arbufte dans un terrein graveleux; il sélève à environ 3 pieds.

\section{Vaccinium mucronatum.}

Cet arbufte acquiert 2 pieds de hant; fes fruits font bons à manger; il vient dans un terrein ftérile.

\section{$V$ acsinium multiflorum.}

C'eft un très-bel arbuifte à fleurs; il croît de deurx خे 4 pieds de haut dans un fol graveleux, \& doit être envoyé en plant.

$$
\text { Vaccinium, nova species. }
$$

Ceft un fort bel arbriffeau à fleurs; il a depuis 2 jufqu'à $s$ pieds de haut.

$V$ accinium ovale, nova fpecies.

Cet arbutte a de très-beiles flcurs; il a depuis 2 jufqu'à 5 pieds de haut, dans un terrein graveleux; il faut l'envoyur en plant.

$$
\text { I'aciinium oxycocus. }
$$

Cet a briffeau a cnviron 2 dे 3 picds de hauteur; ellc ranipe if $l_{a}$ terre, fos fruits font aigres, \& on s'en fert pour faire du tartre; elle croit dans un fol hunide \& fablonneux, fijet de être inondé une partie de laanée; on doit l'envoyer en plant. 
Vaccinium ftrictum, rubrum.

Cet arbufte a environ 2 pieds de haut; il aime un fol ftérile \& doit être envoyé en plant.

$V$ accinium rubrum, acidum.

Cet arbufte a environ 4 pieds de haut; fes fleurs font jolies \& aigres ainfi que fes feuilles \& bonnes manger; il croît dans un fol humide \& argilleux, \& doit être envoyé en plant.

\section{Viburnum acuminatum.}

Cet arbufte a 8 pieds de haut; fes fleurs font belles, fon bois compact \& il croit dans un fol fablonneux.

\section{$V$ iburnum aquaticum.}

Cet arbre a 4 pieds de haut; fon bois eft compact; il croît dans un fol humide.

\section{Viburnum glabrum, majus.}

Cet arbufte a de très-belles fleurs; il s'élève de 4 aे 8 pieds de haut.

Viburnum glabrum, minus.

Cet arbufte a environ 4 pieds de haut; il a de jolies fleurs \& vient dans un terrein pierreux.

Viburnum prunifolium.

Cet arbufte porte de très-belles fleurs; il s'élève depuis 8 jufquà 20 pieds; c'eft le meilleur arbufte que je connoiffe pour des hayes; il croît fur touté fortes de fols; on mange fes bayes.

$V$ itis Americana, dulcis.

Elle donne le raifin le plus agréable de tous ceux qui font natifs d'Amérique; fes graines font petites, 
mais je penfe quion pourroit cultiver cette efpèce de vigne dans ce pays-ci; elle croît fur toutes fortes de fols.

\section{$V$ itis procumbens.}

Cette vigne n'eft pas très-haute, \& fouvent rampe fur la terre \& grimpe fur de petits arbuftes; fon fruit eft aigre \& n'a pas un goût très-agréable; elle croît fur un fol ftérile.

\section{Vitis Virginica.}

C'eft, dans mon opinion, le meilleur de tous les railins Américains; il pzut être cultivé avec beaucoup d'avantage en Amérique pour en faire du vin; il croit dans toutes fortes de lols.

\section{Vitis vulpina.}

C'eft une vigne qui produit des raifins; elle monte autour des arbres à une grande hauteur; elle aime un fol riche \& humide.

$$
V \text { itis vulpina, alba. }
$$

C'eft urie des efpèces de la vigne précédente; fon fruit eft bien \& beaucoup plus doux; la vigne ne devient pas auffi. forte; elle aime un fol riche \& hunide.

$$
\text { Zanthoxylon, clava herculis. }
$$

Cet arbufte a Is pieds de haut; il croît fur toutes fortes de fols; fes racines font médicinales; il doit être envoyé en plant.

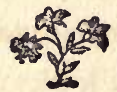




\section{PLANTES HERBACEES.}

\section{Actea racemofa.}

Elle a 6 pieds de haut, eft très-jolie quand elle eft en fleurs, aime un fol riche $\&$ léger $\&$ de l'ombre.

Adiantum pedatum.

Ceft une très-belle plante; elle a environ I pied de haut, croît dans un fol riche \& léger \& 2 l'ombre; $\&$ doit être envoyée en plant.

\section{Agrimonia major.}

Elle a environ 2. pieds de haut, \& croît dans un fol léger.

$$
\text { Agrimonia ruftica. }
$$

Elle a environ 3 pieds de haut, \& croît dans un fol riche \& léger.

$$
\text { Agrimonia trifoliata, nova Species. }
$$

Elle a environ 3 pieds de haut; elle aime un fol riche \& léger \& l'ombre; elle eft médicinale.

\section{Agrimonia vulgaris.}

Elle a environ un pied de haut, eft médicinale \& croît dans toutes fortes de fols.

$$
\text { Allium latifolium, nova fpecies. }
$$

Elle a environ 5 potices de haut; c'ct une forte plante bulbeufe, fes feuilles font grandes; elie croit: dans un fol riche \& fablonneux, \& doit être envoye en plant. 


\section{(29)}

\section{Anemone thalictroides:}

Cette jolie plante a fleurs croît à environ 4 ponces, Heurit en Avril, aime un fol riche \& léger \& l'ombre. On m'a appris que les racines de cette plante guériffent les morfures de ferpent; il y a une variéte de cette efpèce avec des fleurs doubles, qui eft très-belle.

Angelica alba, nova Species.

Cette plante très-élégante a fix pieds de haut, \& aime un fol léger \& riche.

Angelica Americana, major, nova Species.

Elle a 5 pieds de haut, eft fort belle, fleurit en Juillet, aime un fol léger \& ftérile; elle eft médicinale. Angelica Americana, minor, nova Species.

Elle a deux pieds de haut, eft jolie, fleurit en Mais \&s Juin, aime un fol léger \& l'ombre.

\section{Angelica aquatica.}

Elle a environ 6 pieds de haut, fleurit en Juin \& Juillet, eft médicinale, croît dans un fol riche \& humide.

Angelica paftinaca, nova Species.

Elle a 5 pieds de haut \& croît dans un fol marécageux.

$$
\text { Apocynum androsemifolium. }
$$

Elle a environ 3 pieds de haut; fes fleurs font plus belles que celles de toutes les autres efpèces d'apocyns; .elle fleurit en Août, aime un fol riche \& léger avec l'ombre, \& doit être envoyé en plant. 
(30)

Apocynum cannabinum.

Elle a depuis I jufqu'à 3 pieds de haut; fes fleurs font jolies; elle croît fur toutes fortes de fols, \& fleurit en Juin \& Juillet.

\section{Apocynum majus.}

Elle a environ 5 pieds de haut, de belles fleurs, croit dans un fol fablonneux \& fleurit en Juillet.

$$
\text { Aquilegia Canadenfis. }
$$

C'eft une très-élégante plante à Hleur; elle a environ I pied de haut; croît dans les fentes des rochers, \& vient fur toutes fortes de fols; elle fleurit en Mai, Juin \& Juillet.

\section{Aralia herbacea.}

Elle a 6 pieds de haut, eft médicinale, croît dans un fol riche \& léger.

\section{Arum majus.}

Elle a environ I pied de haut, eft médicinale, fleurit en-Mai \& aime un fol riche \& humide.

\section{Arum dracontium.}

Elle a environ I pied de haut, fleurit en Avril, eft médicinale, croît dans un fol riche \& humide parmi des roches.

$$
\text { Arum Virginicum. }
$$

Les feuilles de cette plante font fort grandes; elle eft médicinale, fleurit en Avril ; eile cilt curieufe \& croît dans un fol riche \& humide.

$$
\text { Afarum Canadenfe. }
$$

Elle ficuiit en Mai ; fes fleurs font curieufes; elle croît dans un fol léger, \& elle eft médicinale. 


\section{(3I) \\ Afarum Virginicum.}

Ceft une belle plante; fes fenilles font dune odeur agréable; elle croit dans un fol riche \& léger, \& elle eft toujours verte.

$$
\text { Afclepias flore rubro. }
$$

Elle a environ 2 pieds de haut $\&$ de belles fleurs; elle fleurit en Juillet, aime un fol riche \& léger. \& Pombre.

$$
\text { Afclepias foliis villofis: }
$$

Elle a environ 2 pieds de hant de très-belles fleurs; des qualités médicinales; elle croît dans un fol fablonneux \& ftérile, fleurit en Juillet \& doit êtré envoyé en plant.

\section{Afclepias incarnata:}

Elle a environ 2 pieds de hawt, de belles fleurs; croît dans un fol fablonneux \& humide, \& fleurit en Aoutt.

$$
\text { Afplenium rhizophyllum. }
$$

Ceft une plante curieufe; elle croît dans des fentes \& fur le fommet des rochers; elle eft toujours verte \& doit être envoyée en plant. Ce qui ma engagé a comprendre dans le catalogue cette plante, ceeft qu'elle peut être recherchée par des curieux.

$$
\text { After aculeatus. }
$$

C'ét: une belle plante; elle a deux pieds de haut, fleurit en Septembre, croît dans un fol fablonneux \& Itérile, \& doit être envoyée en plant.

$$
\text { After aquaticus, major, nova species. }
$$

Elle a 8 pieds de haut; $c^{\prime}$ 'ft une belle plante quand 


\section{(32)}

elle eft en fleurs, ce qui arrive en Septembre; ello croît fur un fol riche \& humide.

$$
\text { After corymbosus. }
$$

Cette plante a I pied de.haut, eft jolie en fleurs; Heurit en Août, vient fur un fol ftérile \& graveleux.

$$
\text { After cordatis foliis. }
$$

Elle a de belles fleurs, 4 pieds de haut environ, fleurit en Septembre, aime un fol riche \& léger \& l'ombre.

$$
\text { After cordatis foliis, nova Species. }
$$

Elle eft très-belle quand elle eft en fleurs; elle croît dans un fol ftérile \& fablonneux \& fleurit en Septembre.

$$
\text { After foliis herfutis, nova Species. }
$$

Elle a environ 3 pieds de haut, fleurit en Septem. bre, croît fur toutes fortes de fols, pourvu quuil foit riche.

$$
\text { After grandiflorus. }
$$

Elle a 5 pieds de haut; fes fleurs font belles, croitt dans un fol riche \& léger \& dans l'ombre.

\section{Calceolus acuminatus.}

Elle a environ I pied de haut, fleurit en Mai; elle eft curieufe \& fes fleurs font jaunes; clle aime un fol riche \& léger; elle doit être envoyée en plant.

\section{Calceolus latifolius.}

Elle a environ $S$ pouces de haut, fleurit en Mai ; 1es fleurs font curieufes \& rouges, elle aime un fol fablonneux \& l'ombre 3 elle doit être envoyée en plant. 
Capraria cordato folio:

Elle a 8 pieds de haut, monte autour des arbufies; croît dans un fol riche \& humide.

\section{Carum Americanum.}

Elle a 3 pieds de haut \& croît dans un fol léger.

Carum aquaticum.

Plante curieufe \& médicinale, qui croît à environ 2 pieds de haut dans un fol riche $\&$ humide.

Calja chamocrifta.

Elle a environ 4 pieds de haut; fes fleurs font citrieufes, mais fes feuilles ont une odeur défagréable; elle fleurit en Août \& aime un fol fablonneux.

Calia procumbens.

Elle a de belles fleurs, fleurit en Juillet \& Août; elle paroît éprouver une vive fenfation quand on la touche; elle croît dans un fol riche \& humide.

$$
\text { Cafja fricza. }
$$

Elle eft annuelle, a environ 7 pouces de haut; c'eft une jolie plante; elle fleurit en Juillet \& aime un fol léger.

$$
\text { Clematis virginica. }
$$

Elle a une jolie fleur, monte autour des buiffons à la hauteur de 6 pieds, flêurit en Août, aime un fol riche \& léger.

Convallaria major.

C'elt une luperbe plante; elle a 5 pieds de haut, croît dans un fol hum: ace \& fablonneux, fleurit en Juin \& doit être envoyée en plant. 


\section{(34)}

\section{Convalloria raumosa.}

Elle a environ 2 pieds de haut; elle a de fort belles fleurs, aime un fol riche \& léger, \& l'ombre, \& fleurit en Mai.

\section{Coreopfis latifolia.}

Elle a environ 3 pieds de haut; elle eft belle, Aleurit pendant 3 mois \& croît fur toutes fortes de fols.

\section{Cypripedium Americanum.}

Elle a 5 pieds de haut, des fleurs curieufes, fleurit en Août; elle eft annuelle, aime un fol riche \& humide.

$$
\text { Digitalis Americana, nova Species. }
$$

Elle a environ 5 pieds de haut, de grandes \& belles fleurs jaunes; elle fleurit en Août, aime un fol gra* veleux \& P'ombre.

Digitalis flore rubro, nova Species.

Elle a environ I pied de haut; c'eft une des plus belles plantes a fleur de l'Amérique.

$$
\text { Digitalis lyrata, nova jpecies. }
$$

C'eft une délicicufe plante à fleurs; elle eft annuelle, croît dans un fol ftérile, \&’ fleurit en Septembre.

$$
\text { Dionea muscipula. }
$$

Cette plante curieufe ma été d'abord connue par le compte que m'en rendirent quelques - uns de mes amis en 1763 : quelques années après, ayant été envoyé par Sa Majefté Britannique en Amérique, pour y rafembler des plantes nouvelles \& curieufes, je srouvai selle-ci en grande abondance dans la Caroline 
cu Nord \& dans quelques parties de celles du Sud, d'où jen apportai plufieurs à mon retour en Europe; clles crurcint dans mon jardin près d'Illfwerth, \& fleuriffent avcc leur vertu fenlitive. Ce fut d'après ces plantes, que M. Ellis de la fociété de Londres, fit deffiner la Muffipala, \& en donna une defcription en 1780. Les femences de cette plante exigent un traitement particulier. Il faut la femer dans un pot dont la terre foit compofé de mouffe de marais, coupée en brins d'environ I pouce de long, \& bien mêlée avec une terre riche \& fablonneufe. Il faut laiffer au haut du pot I pouce de profondeur, qui foit rempli en entier d'un fable riche dans lequel on met les femences, $\&$ mettre le pot dans une terrine tenue toujours pleine d'eau. On la doit femer en Mai, mettre le pot à l'ombre, \& ne point la troubler pendant plufieurs années, car les graines ne montent point la premiere année. Il faut avoir foin de la purger de mauvailes herbes, ce pot doit être placé en hiver dans une ferre: c'elt par cette méthode: que je fuis parvenu à en élever plufieurs plants; mais la meilleure maniere de s'en procurer en Europe, eft de fe les faire envoyer en plant. La grande dépenfe quill faut faire pour s'en procurer; eft hors de mon pouvoir.

\section{Erigeron Philadelphium.}

Cette plante a de belles fleurs, elle croît fur un fol atérile d'un à deux pieds; elle eft en fleurs en Septembre.

\section{An Evonymus? planta nova.}

Cette plante a 3 pieds; je ne lai jamais vu en fleur, quoique femé de bonne heure; elle croît dans un fol pierreux.

\section{Eupatorium fore albo.}

Elle a deux pieds de haut, c'elt une belle plante, elle fleurit en Septembre, aime un foll'́ger \& l'ombre. 


\section{(36)}

Eupatorium Vinginicum.

Ceft une jolie plante a fleur; elle fleurit en Sep. tembre \& croît fur un fol léger.

Euphorbia lincaribus foliis, nova Jpecies.

Ceft une jolie plante; elle croît-dे environ 2 pieds dans un fol léger \& Aleurit cn Juin.

\section{Geranium virginianum.}

Elle a environ I pied de haut, a de jolies fleurs \& croît fur toutes fortes de terreins.

Gentiana amminata, nova pecies.

Elle croît dans un fol humide, a des jolies fleurs, fleurit en Octobre \& doit être envoyée en plant.

$$
\text { Gentiana alba, nova Species. }
$$

Cette plante a environ I pied de' haut, fes racines font longues \& épaifies; c'eft un excellent amer, il croît dans un fol léger, doit être envoyé en plant \& fleurit en Septembre.

\section{Gentiana imperialis. (Lée.)}

Elle a ? pieds de haut; fes fleurs font belles; elle vient dans un fol humide \& argilleux, fleurit en Octobre; elle eft médicinale \& doit être envoyée en plant.

\section{Gramen Americanum.}

Cette herbe vient fort haute fur un fol léger; elle donne une belle pâture pour le bétail ; elle eft annuelle.

\section{Gramen canarinum.}

Ce fourage peut être cultivé avec avantage, donne du bon foin, croît dans un fol fablonneux, \& ilef: annuel. 


\section{(37)}

Gramen lancolatis foliis, majus, nova Species?

Cette herbe, qui pent devenir d'un ufage univerfel, donne du bon fourage \& croit dans un fol Itérile; elle peut être cultivée dans des terres incultes en Europe; elle eft vivace.

\section{Gramen lycurum.}

Ce fourrage peut être cultivé avec avantage dans in fol fablonneux, mais il eft annuel.

\section{Gramen medium, nova Species.}

Cette herbe donne un fort bon foin; elle croît dans un fol ftérile, eft vivace; cette efpèce \& le lancolatis foliis font deux efpèces d'herbes qui peuvent être avantageufes pour convertir les terres les plus ftériles en prairies.

Helianthus latifolius, nova Species.

Elle a environ 8 pieds de haut, des fleurs curieufes; elle fleurit en Aout \& aime un fol riche.

Helianthus multiflorus, nova Species.

Elle a environ' 5 pieds de haut, fleurit en Aocit, \& croit dans un fol riche \& humide.

\section{Helianthus vulgaris, nova Species.}

Elle a environ 8 pieds de haut \& beaucoup de fleurs; elle fleurit en Septembre \& croit fur toutes fortes de fols.

\section{Helonias afphodeloides.}

C'eft une plante curieufe, elle a deux pieds de haut, fleurit en Juillet, aime un fol fablonneux, \&6 çoit être envoyée en plant. 


\section{(38) \\ Helonias bullaza.}

Elle $\alpha$ environ 2 pieds de haut 3 fa fleur eft trèsbelle; elle fleurit en Mai, aime un fol humide \& doit être envoyée en plant.

\section{Helonias marylandica.}

Cette plante eft curieufe en Botanique; elle a 2 pieds de haut, fleurit en Juillet, aime un fol argilleux \& humide, \& doit être envoyée en plant.

$$
\text { Helxine cordata, nova species. }
$$

Cette plante grimpante eft longue de 12 pieds; monte autour des arbuftes ou rampe fur la terre; elle peut ètre cultivée avec avantage en confidération de fes graines, qui font utiles aux hormcs \& au bé. tail; elle eft vivace \& croît fur toutes fortes de fols.

\section{Hieracium latifolium.}

Elle croît à 2 pieds de haut fur un fol argilleux.

$$
\text { Hordeum, nova Species. }
$$

Elle a environ 2 pieds de haut c'eft le feul orge connu qui foit vivace; je ne doute pas quil ne puiffe gagner beaucoup par la culture \& devenir d'un grand ufage; on le trouve parmi des rochers \& des pierres, dans quelques parties des montagnes du Nord en Virginie.

$$
\text { Hydraftis canadenfis. }
$$

Elle a environ I pied de haut; fes racines font d'une belle couleur jaune; elle croît dans un fol riche \& léger, \& doit être envoyée en plant.

Hypoxis erecta.

C'eft une belle petite plante; elle a environ 5 poucès de haut, fleurit en Juillet \& Août, croît fur 


\section{(39)}

un fol argilleux \& humide, \& doit ếtre envoyée en plant.

\section{Lilium canadenfe.}

Il a 4 pieds de haut, de belles fleurs, fleurit en Juin \& Juillet, croît dans un fol humide \& fablon neux, doit être envoyé en plant.

\section{Lilium grandiferum.}

C'eft une plante a fleurs charmantes; elle a io pieds de haut; elle eft beaucoup plis belle que le Lilium Superbum; elle croît dans un fol léger \& fleurit en Juin \& Juillet.

\section{Lilium Superbum.}

Elle a 10 pieds de haut, fleurit en Juillet \& Août ; elle eft très-belle; elle a fouvent 30 fleurs fur une tige; croît dans un fol riche $\&$ humide, \& doit être envoyée en plant.

\section{Lilium vulgare.}

Il a environ I pied de haut, de belles fletrrs; fleurit en Mai, croît fur un fol ftérile.

\section{Linum Virginianum.}

Elle a environ 2 pieds de haut, \& je penfe qu' elle peut être cultivée avec avantage en Amérique \& en Europe.

\section{Lobelia cardinalis.}

Elle a environ 2 pieds de haut, fleurit en Août; c'eft une belle plante à fleurs; elle croît dans un fol riche \& humide; elle doit être envoyée en plant.

\section{Lobelia kalmia.}

Elle a environ 2 pieds de haut, fleurit en Aouit \& Septembre; on dit qu'elle poffede beaucoup de qua: lites médicinales; elle drit être envoyée en plant.

$$
\text { C iv }
$$




\section{(40)}

\section{Lobelia lincaribus foliis:}

Elle a environ 2 pieds de haut, de jolies fleurs: croît fur un fol léger a l'ombre; ceet une plante an. nuelle.

\section{Lobelia nudo flore, nova species.}

Elle croit fur toutes fortes de fols, eft annuelle; Geurit en Septembre, \& a environ 6 pieds de haut.

\section{Lupinus hirfutus.}

Ceft une belle plante a fleurs; elle fleurit en Juin: aime un fol léger \& ftérile, croît à un pied de haut, \& doit être envoyée en plant.

\section{Lupinus Virginicus.}

Ceft une belle plante a fleurs; elle a environ un pied de haut, fleurit en Avril \& Mai, \& aime un fol Cablonneux.

\section{Lycopodium acerofum, nova Species.}

Elle a envison 4 pouces de haut; elle vient dans les lieux humides \& a l'ombre; c'eft une plante fort belle \& toujours verte.

\section{Lycopodium complanatum.}

Elle vient quelquefois à dix picds de hautcur; ceeft une plante élégante \& toujours verte; elle croît dans un fol humide, ftérile \& argilleux, \& doit être envoyée en plant.

\section{Lycopodium obfcurum.}

Elle vient dans un fol graveleux \& a l'ombre; elle a environ fix pouces de haut; elle eft trìs-belle, toujours verte, \& doit être envoyée en plant.

\section{Medeola Virginica.}

Elle a environ un pied de haut, fleurit en Juin; 
celt une plante curieufe; elle aime un fol riche. humide, \& doit être envoyée en plant.

$$
\text { Mentha hirfuta. }
$$

Elle a environ un pied de haut, fleurit en 'Juillet, \& croît dans un fol léger.

\section{Menthalanceolatis foliis, flore corymbofo.}

Elle a 3 pieds de haut, fes feuilles ont une odeur agréable; elle fleurit en Juin, Juillet \& Août; elle êt médicinale; \& croît fur un terrein ftérile.

$$
\text { Mentha Marylandica. }
$$

Elle a environ deux pieds de haut, eft médicinale, \& croît dans un fol léger.

\section{Mitchella repens.}

Ceft une plante botanique curieufe; fes fruits font d'une belle couleur ronge; elle fleurit en Juin, croît dans un fol humide \& ftérile, \& doit être envoyée en plant.

\section{Nolinia.}

C'eft un nouveau genre de plante, que jappellerai Nolinia, en l'honncur de M. P'Abbé Nolin. Elle croît d environ 10 pieds de haut, dans la forme d'une vigne, monte autour des arbres \& des arbuftes, a des fleurs mâles \& femelles fur des plantes féparécs, aime un fol riche \& humide, \& doit être envoyée cn plant.

\section{Ocymum Americanum.}

Elle exhale une odeur agréable, a de jolies fieurs, croît à environ un pied de haut, aime ua fol léger \& fablonneux, \& eft amnuelle.

Orchis acuminata, nova species.

Elle a environ quatre pouces de haut, de jolies 
(42)

fieurs, \& fleurit en Juillet; elle croít dans des forndrieres, \& doit être envoyée en plant.

\section{Orchis foliis lanceolatis, nova $\int p e c i e s$.}

Elle a environ un pied de haut; fes fleurs font belles \& fleuriffent en Août; elle crôit dans un fol marécageux, \& doit être envoyée en plant.

Orchis laiffolia minor, nova Species.

Ceft une plante curieufe; elle a environ 4 pouces de haut, fleurit en Août, aime un fol léger \& l'ombre, \& doit être envoyée en plant.

$$
\text { Orchis spicata, nova Species. }
$$

Ses fleurs font belles \& curicufes; elle fleurit en Août, \& doit êtré envoyée en plant.

\section{Origanum Virginianum.}

Elle a environ I pied de haut, eft médicinale, croît dans un fol ftérile \& pierreux 2 doit être envoyée en plant.

$$
\text { Ofmunda cinnamomea. }
$$

Elle a environ 2 pieds de haut; elle croit dans un fol hunide \& argilleux , \& doit être envoyée en plant.

\section{Ofmunda Virginiana.}

Elle a environ I pied de haut; croit dans un fol ferme \& doit être envoyée en plant.

\section{Panax quinque folium.}

Cette plante a environ I pied de haut ; fa racine eft en grande réputation pour fes qualités médicinales; elle aime un fol riche \& léger, \& fleurit en Juin.

$$
\text { Phyrolaced decandra. }
$$

Cette plante a 10 pieds de haut ; clle croît dans 


\section{(43)}

un fol riche \& humide; fes fruits donnent une belle tointure rouge. Les jeunes pouffées font bonnes a manger, mais il faut que la plante ait au moins 3 ans pour que les racines jettent de nouvelles pouffés; beaucoup de gens en Amérique la préferent aux afperges; mais, lorfquelle eft vieille, elle a une qualité dangereufe; les racines de cette plante fervent dans la cure des chancres obftinés, àu moins les Indiens en font ufage dans cette maladie.

\section{Podophyllon peltatum.}

Elle a environ I pied de haut, de belles fleurs, un fruit agréable'à manger; elle aime un fol riche \& humide avec de l'ombre; elle fleurit en Mai.

\section{Polypodium aquaticum, nova Species.}

Elle a environ 3 pieds \& n'eft pas toujours verte, mais dans l'été elle a une fort belle apparence; elle doit être envoyée en plant.

\section{Polypodium majus.}

Elle a environ 6 pieds de haut; elle eft la plus grande de toutes les efpèces dans l'Amérique Septentrionale; je n'ai jamais vu fa fructification; elle croît dans un fol riche \& humide, \& doit être envoyée en plant.

\section{Polypodium virginicum.}

Cette petite plante a environ 5 pouces de haut; elle eft toujours verte, croît dans un fol riche fur le fommet des rocs; elle doit être envoyée en plant.

$$
\text { Polypodium vulgare. }
$$

C'eft une belle fougere toujours verte; elle croît dans un fol pierreux a $l_{a}$ hauteur de 2 pieds; elle doit être envoyée en plant. 


\section{(44) \\ Pontederia cordato folio.}

Ceft une belle plante à fleurs; elle a environ 3 pieds de haut, Heurit en Juillet \& Août, croît dans un terrein riche \& bien arrofé, \& doit être envoyée en plant.

\section{Prenanthes altifima.}

Elle a environ 4 pieds de haut, fleurit en Octobre, aime un fol argilleux.

\section{Pulmonaria canadenfis.}

Elle a environ 2 pieds de haut, de belles fleurs; elle fleurit en Avril, aime un fol riche \& fablonneux, $\&$ doit être envoyée en plant.

\section{Pyrola maculata.}

Elle a environ 4 poitces de haut, eft toujours serte; fleurit en Juillet, fes fleurs font belles \& d'une odeur agréable; elle croît dans un fol riche \& léger \& $\lambda$ l'ombre; elle eft trop lente à venir de graine, \& doit être envoyée en plant.

\section{Pyrola umbellata.}

Elle a environ 5 pouces de haut; fes fleurs fons fouvent d'une couleur de pourpre pâle; c'eft tune plante vivace toujours verte; elle aime un fol graveleux \& l'ombre; elle doit être envoyée en plant.

\section{Rhexia minor.}

C'eft une belle petite plante à fleurs; elle a environ 7 pouces, fleurit en Août, aime un fol humide \& argilleux, \& doit être envoyée en plant.

\section{Rhimanthus Virginicus.}

Elle a environ 4 pieds de haut, n'a qu' une fleur, eft médicinale, croît fur un fol riche \& leger, \& doit être' envoyée en plant: 


\section{(45)}

\section{Rapuntium non Spinofum.}

C'eft une plante fucculente; clle a de belles flcurs, crôt dans un fol fablonncux, fleurit en Août.

\section{Rubia americana.}

Elle a environ I pied de haut, fert dans la teinture du rouge; c'eft elle dont les Indiens d'Amérique font cette couleur rouge, dont ils peignent leurs cheveux \& qui ne paffe jamais; elle croît dans un fol ftérile; fes racines \& la partic qu’on emploie dans la teinture font vivaces.

\section{Rudbeckia hirta.}

Elle a environ 2 pieds de haut, de belles fleurs; elle fleurit en. Septembre, aime un fol argilleux \& humide.

\section{Sanguinaria cartadenfis.}

Elle a environ 5 pouces de haut, fleurit en Avril; ceft une belle plante; elle aime un fol riche \& léger \& l'ombre; \& doit être envoyée en plant.

\section{Sarracenia purpurea.}

Ceft une plante très - étonnante; elle fleurit en Juin; croît dans un fol marécageux; les tiges de fes fleurs ont environ I pied de haut; les feuilles font creufes de la figure d'une burette, dont le pied repofe fur Ia terre, \& elles font ordinairement remplies d'eau; elle doit être envoyée en plant.

\section{Sedum procumbens.}

Elle eft fort épanouic, fleurit en Avril, croît fur un terrein humide \& fablonneux, \& doit être envoyée en plant.

Serratula prealta.

Elle a environ $s$ pieds de haut; eft belle quand 
elle eft en fleurs; aime un fol piche \& léger, \& fleurit en Juin.

\section{Sideritis acuminata.}

Elle a environ 3 pieds de haut; fleurit en Août; aime un fol riche \& humide.

\section{Sideritis major.}

Celt une plante majeltueufe de ro pieds de haut; qui a une belle apparence quand elle eft en fleurs, ce qui arrive en Septembre; elle aime à croitre dans un lol riche \& léger.

\section{Solidago nudo fiore?}

Elle a environ I pied de haut, de belles fleurs; elle eft médicinale, theurit en Septembre; croît fur un fol ftérile.

$$
\text { Solidago dulcis. }
$$

Elle a 3 pieds de haut, fes feuilles ont une odeur agréable, fes fleurs funt jaunes; elle fleurit en Septembre; elle aime un fol fablonneux.

\section{Solidago flore albo, nova species.}

Elle a environ 2 pieds de haut, fes fleurs font blanches \& jolies; elle eft en grande réputation parmi les Sauvages, pour fes vertus médicinales; elle fleurit en Septembre, croît dans un fol léger \& à P'ombre.

$$
\text { Solidago flore alzernato. }
$$

Elle croît à la hauteur de 3 pieds; elle eft belle quand elle eft en fleurs, eft médicinale; elle croît dans un fol léger \& fitué à l'ombre, \& fleurit en Septembre.

\section{Solidago marina.}

Ceft une plante majeftueufe; elle a 8 pieds; fes fleurs font belles; cllc aime un fol riche \& fablonneux, \& Aleurit en Septembre. 


\section{(47)}

Solidago pratenfis, major.

Elle a I pied de haut, de belles fleurs; fa tige eft d'une belle couleur rouge; elle fleurit en Août.

\section{Solidago Serratifolia.}

Elle a environ 5 pieds de haut, fleurit en Septembre; c'eft ane jolie plante.

Symphytum Americanum, nova species.

Elle a 3 pieds de haut, aime un fol riche \& léger, \& l'ombre; elle eft médicinale.

Symphytum minus, nova Species.

Elle a environ 9 pouces de hatit, fleurit en Mai, eft belle \& croit dans un fol léger.

\section{Triofteum floribus verticillatis.}

Elle a 2 pieds de haut, eft médicinale; elle aime un fol léger.

\section{Veronica Virginica.}

Elle a environ 5 pieds de haut, eft médicinale \& en grande eftime parmi les Indiens pour la guérifon des flux de fang; elle croit dans un fol riche \& doit être envoyée en plant.

$$
\text { Vicia hirfuta. }
$$

Elle eft vivace; a 4 pieds de haut; vient dans un fol humide \& riche; a de belles fleurs \& fleurit en Août.

$$
\text { Vicia marylandica. }
$$

Elle a 5 pieds de haut; croît dans un fol ftérile \& elle eft annuelle. 
(48)

Vicia fricza, nova Specie:

Elle a 4 pieds de haut, fleurit en Août \& Sep tembre; elle eft belle \& aime un fol graveleux.

\section{$V$ icia trifoliata, nova Species.}

Elle s'elève $\mathrm{d}$ io pieds de haut, monte autour des arbuftes, \& peut être cultivée avec quelquavantage pour la nourriture du bétail; elle crôt dans un fol léger \& elle eft vivace.

Viola cordato folis, nova $\int p e c i e s$.

Elle a environ 6 pouces de haut; elle fleurit en Mai; crôt dans un fol riche \& léger, \& à l'ombre.

$$
\text { Viola foliis palmatis. }
$$

Ceft une belle petite plante qui a environ 4 pouces de haut; elle fleurit en Mai ; elle croît dans un fol léger \& ftérile, \& doit envoyée en plant.

$$
\text { Yucca filamento } a \text {. }
$$

Ceft une plante curieufe; fes filaments fervent a faire des cordes qui font étonnamment fortes; elle a de belles fleurs \& fleurit en Juillet \& Août dans un fol léger \& fablonneux. 
Lifte des Arbres, Arbrifleaux \& Plantes qu'on ne peut fe procurer que par des voyages difpendieux dans le continent de l'Amérique, Ė que $M$. Yong n'a point encore élévés en affez grand nombre pour. les envoyer en Europe.

\section{ARBRES ET ARBUSTES.}

Amorpha fructicosa, qui habite en Caroline.

Cet arbufte 2 environ 6 pieds de haut, de jolies fleurs, \& croît dans un fol léger \& humide.

Andromeda arborea, qui habite en Caroline.

Cet arbre eft haut de 40 pieds; il eft très-bedu \& croît dans un fol léger.

Andromeda latifolia foliis alcernis, qui habite en Caroline.

Ceft un arbufte élégant, toujours vert; il a environ 5 pieds de haut; fes fleurs font couleur de pourpre \& d'une odeur agréable.

Annona grabra, qui habite en Caroline.

Cet arbre a environ 20 pieds de haut; fon écorce eft dure, \& peut être manufacturéce en fortes cordes; il croît dans un fol riche \& humide.

Annona muricaza, qui habite en Virginie.

Cet arbre a environ 25 pieds de haut; fon écorce eft fort dure \& fon fruit bon à manger; il croît dans iun fol riche \& léger. 


\section{(so)}

\section{Annona triloba, qui habite en Caroline.}

Cet arbre a environ 20 pieds de haut; fon écorce eft dure, on en fait des cordes; il croît dans un fol riche \& humide, \& fon fruit eft bon a manger.

Bignonia foliis fimplicibus, qui habite en Caroline.

Cette plante eft la gloire de la Caroline; elle parfume les bois de l'odeur la plus délicieufe; 8 pour une ame romanefque, elle change les déferts les plus fauvages en paradis; elle fleurit en Avril \& Mai, croît dans un fol riche, léger \& humide, a la hauteur de 20 pieds; elle monte autour des arbres \& des arbriffeaux. Calycanthus lancolatus, qui habite en Caroline.

Cet arbufte a environ 6 pieds de haut; il aime un fol riche \& léger.

Calycanthus latifolius, qui habite en Caroline:

Cet arbufte a environ 4 pieds de haut, eft en fleurs une grande partie de l'été; il a une odeur très-agréable, eft très-beau \& croît dans un fol léger.

Cafine foliis lancolatis, alternis, qui habite en Caroline.

Cet arbufte a environ 10 pieds de haut, croît dans un terrein fablonneux; il eft toujours vert \& on en fait un thé fort fain.

Caffine d'ahoon, qui habite en Caroline.

Cet arbufte a environ 15 pieds de haut; il croît dans un fol léger; il eft toujours verd \& joli.

Cuprefus difticha, qui habite en Caroline $\&$ en Virginie.

Cet arbse croît a la bauteur de 70 \& 80 pieds; il 
eft d'une groffeur étonnante; cet arbre \& le Liriodendrom font les plus grands arbres de toute l'A. mérique, \& je crois de tout le monde; il croît dans un fol riche \& humide.

\section{Efculus Pavia, qui habite en Caroline.}

Cet arbufte a environ 12 pieds de haut, de belles fleurs, fleurit dans le mois de Mai \& de Juin \& croît dans un fol léger:

\section{Gordonia, Ellis hypericum, qui habite en Caroline.}

C'eft un très-bel arbre toujours vert; il s'élève a 70 pieds de haut \& croît dans un fol riche \& liumide. Gordonia pumila, deriduis foliis, qui habire en Floride.

Ce grand arbufte a environ 20 pieds de haut; il a été découvert par M. William Bartram; je l'ai vus en fleurs le mois d'Août $178 I$; il avoit 4 pieds de baut.

Guilandina,.Bonduc, qui habite en Canada.

Cet arbufte a environ 30 pieds de haut \& croît dans un fol léger.

Illicium floridanum, qui habite en Floride.

Cet arbufte a environ 15 picds de haut; il fleurit en Juillet dans la Floride; il eft toujours vert, fes fleurs.ont une odeur agréable; il croît dans un fol riche \& humide.

Laurus Borbonia, qui habite en Caroline \& en Virginie.

Cet arbufte a environ Is pieds de haut, croît dans un fol riche \& humide, \& il eft toujr: vert.

$\mathrm{D}$ ij 
Laurus indica, qui habite en Virginie:

C'eft un laurier toujours vert; il a environ I2 pieds de haut, \& il aime un fol riche \& humide.

Laurus nobilis, qui habite en Caroline.

Cet arbufte vient dans la Caroline a 15 pieds de haut; il eft beau, aime un fol riche \& léger.

Ledum latifolium, qui habite à la baye d'Hudfon.

Cet arbufte a environ 2 pieds de haut; il eft toujours vert, fleurit en Juillet \& croit dans un fol riche \& humide.

Ledum paluftre, qui habite à la baye d'Hudfon.

C'eft un bel arbufte toujours vert; il a environ 2 pieds de haut, croît dans un fol marécageux, \& fleurit en Août.

Magnolia acuminata, qui habite fur les bords
de la rivicre.

Cet arbre a environ 60 pieds de haut ; il eft trèsgros \& croît dans un fol_riche.

Magnolia grandiflora; qui habite en Caroline.

Cet arbre s'élève jufqu'a la hauteur de 60 pieds; il eft très-beau lorfquili cft en fieurs, eft toujours vert, fes fleurs durent deux mois; il aime un fol riche, léger \& humide.

Magnolia tripetala, qui habite en Caroline.

Cet Arbre a environ 2'pieds de haut; il fleurit en Juillet, eft beau \& crnit.dans un fol léger.

\section{Mimofa Carolinenfis.}

C'eft une plante qui rampe fur la terre jufqu'à 6 


\section{(53)}

pieds de diftance de fes racines; elle a une prompte fenfation de toutes fortes de touches, \& elle croît fur un fol fablonneux \& térile.

Myria quercifolia, qui habite en Caroline.

C'ett un arbriffeaul'd'une odeur agréable; il croît a environ 2 pieds de haut; dans un terrein fablonneux \& ftérile.

\section{Olea Americana, qui habite en Caroline.}

Cette efpèce d'olivier croît fur un fol riche \& fablonneux, jufquà la hauteur de 30 pieds; il eft tonjours vert.

\section{Pinus paluftris longifolia.}

Cet arbre a environ 80 pieds de haut, croît vite dans un fol fablonneux; on en fait de bons inâts pour des gros vaiffeaux; c'eft un bon bois de confruction utile, \& les jeunes arbres font très-beaux.

\section{Quercus Carolinienfis.}

Cet arbre a environ 50 pieds de haut, \& 2 pieds de diamètre; fon bois eft durable; il eft toujours vert, \& croît dans un fol fablonneux.

Stewairtia malacodendron, qui habite en Caroline $\&$ en Virginie.

Cet arbre a environ 20 pieds de haut, fleurit en Juillet; il eft très-beau \& croît dans in fol léger.

Styrax foliis ovatis, qui habite en Caroline.

Cet arbriffeau a environ is pieds de haut, de belles fleurs; d'une odeur très-agréable, \& croît dans un fol riche \& humide. 


\section{( 54 )}

\section{Yongfonia, qui habite en Caroline:}

C'eft un très-bel arbufte d fleurs, il a environ 2 pieds de haut; il a été apporté à Londres dans l'année 1769 , \& enfuite envoye au fieur Linnzus par le fieur Fothergill, \& jai appris que le fieur Linnarus lui a donné le nom de Yongfonia ; il fleurit en Mai, \& croît dans un fol riche \& humide.

\section{PLANTES HERBACEES:}

\section{Anthericum ventricosum, nova Species, qui \\ habite en Caroline.}

Cette plante a 4 pouces de haut, des fleurs curieufes; elle eft en fleurs une partie de l'été, c'eft-àdire, le matin de trìs-bonne heure; car fa fleur ne peut pas fupporter le foleil.

\section{Afclepas purpurafcens, qui habite en Caroline.}

Elle a environ 3 pieds de haut, une fuperbe fleur, fleurit en Juillet \& Août \& croît dans un fol riche \& léger.

Chironia latifolia, qui habite en Caroline.

Cettc plante a environ 2 pieds de haut; c'eft une très-belle plante; clle eft en fleurs une grande partie de l'été, \& croît dans un fol riche \& humide.

\section{Euphorbia, qui habite en Caroline.}

Cette plante a environ I pied dc haut; les Indiens $s^{\prime}$ 'en fervent pour opérer des guérifons furprenantes; elle crồt dans un terrein léger \& ftérile. 


\section{(55)}

\section{Sarracenia flava}

Cette plante a environ 18 pouces de haut; c'eft une plante très-curieufe; elle fleurit en Avril, et croît dans un fol humide et fablonneux.

Spigelia latifolia, que habite en Caroline

Elle a environ 1 pied de haut, de belles fleurs, des qualites mèdicinales, fleurit in Aout et crôt dans un fol humide et lèger.

\section{Stilla carolinenfis, nova fpecies}

Ceft une belle plante à fleurs; elle a environ 2 pieds de haut, fleurit en Aout et croit dans un fol fterile et humide.

\section{Vinca lutea, que habite en Caroline}

Cette plante fleurit en Juillet; elle eft belle, et elle croit dans un fol riche et lèger.

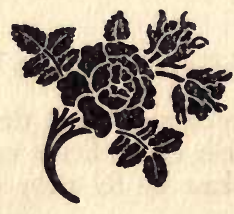





University of Callfornia

$y_{6}$

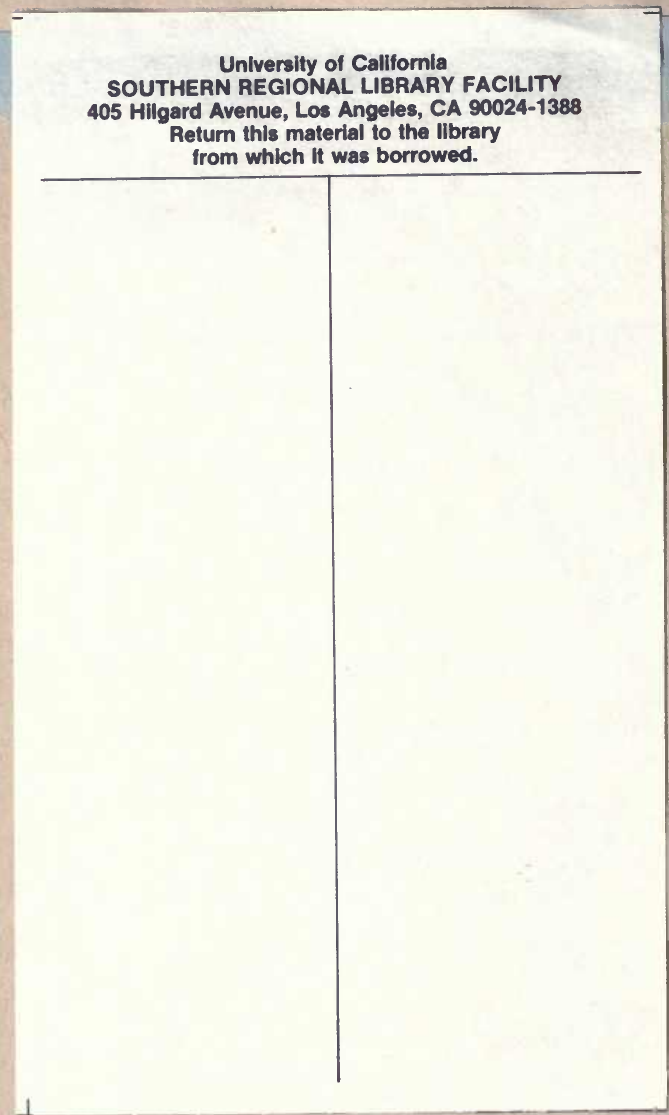




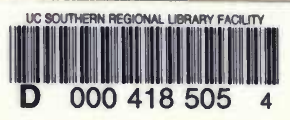


Universi

South

Libr 